\title{
Public T cell receptors confer high-avidity CD4 responses to HIV controllers
}

\author{
Daniela Benati, ${ }^{1}$ Moran Galperin, ${ }^{1}$ Olivier Lambotte, ${ }^{2,3,4,5}$ Stéphanie Gras, ${ }^{6,7}$ Annick Lim, ${ }^{8}$ Madhura Mukhopadhyay, ${ }^{1}$ \\ Alexandre Nouël, ${ }^{1}$ Kristy-Anne Campbell, ${ }^{6}$ Brigitte Lemercier, ${ }^{8}$ Mathieu Claireaux, ${ }^{1}$ Samia Hendou, ${ }^{9}$ Pierre Lechat, ${ }^{10}$ \\ Pierre de Truchis, ${ }^{11}$ Faroudy Boufassa, ${ }^{9}$ Jamie Rossjohn, ${ }^{6,7,12}$ Jean-François Delfraissy, ${ }^{2,3,4}$ \\ Fernando Arenzana-Seisdedos, ${ }^{1,13}$ and Lisa A. Chakrabarti $i^{1,13}$
}

Pasteur Institute, Viral Pathogenesis Unit, Paris, France. INSERM U1184, Center for Immunology of Viral Infections and Autoimmune Diseases, Paris, France. ${ }^{3}$ Assistance Publique - Hôpitaux de Paris (AP-HP), Department of Internal Medicine and Clinical Immunology, University Hospital Paris Sud, Le Kremlin-Bicêtre, France. ${ }^{4}$ Université Paris Sud, UMR1184, Le Kremlin-Bicêtre, France. ${ }^{5}$ CEA, DSV/iMETI, Division of Immuno-Virology, IDMIT, France. Infection and Immunity Program and Department of Biochemistry and Molecular Biology, Biomedicine Discovery Institute, and ${ }^{7}$ Australian Research Council Centre of Excellence for Advanced Molecular Imaging, Monash University, Clayton, Victoria, Australia. ${ }^{8}$ Pasteur Institute, Department of Immunology, Paris, France. ${ }^{9}$ INSERM U1018, Center for Research in Epidemiology and Population Health (CESP), Le Kremlin-Bicêtre, France. ${ }^{10}$ Pasteur Institute, Genomic Bioanalysis Group, Paris, France. "Raymond Poincaré Hospital, AP-HP, Infectious and Tropical Diseases Department, Garches, France. ${ }^{2}$ Institute of Infection and Immunity, School of Medicine, Cardiff University, Cardiff, Wales, United Kingdom. ${ }^{3}$ INSERM U1108, Paris, France.

\begin{abstract}
The rare patients who are able to spontaneously control HIV replication in the absence of therapy show signs of a particularly efficient cellular immune response. To identify the molecular determinants that underlie this response, we characterized the T cell receptor (TCR) repertoire directed at Gag293, the most immunoprevalent CD4 epitope in the HIV-1 capsid. HIV controllers from the ANRS CODEX cohort showed a highly skewed TCR repertoire that was characterized by a predominance of TRAV24 and TRBV2 variable genes, shared CDR3 motifs, and a high frequency of public clonotypes. The most prevalent public clonotypes generated TCRs with affinities at the higher end of values reported for naturally occurring TCRs. The high-affinity Gag293-specific TCRs were cross-restricted by up to 5 distinct HLA-DR alleles, accounting for the expression of these TCRs in HIV controllers of diverse genetic backgrounds. Transfer of these TCRs to healthy donor CD4+ T cells conferred high antigen sensitivity and polyfunctionality, thus recapitulating key features of the controller CD4 response. Transfer of a high-affinity Gag293-specific TCR also redirected CD8 ${ }^{+}$T cells to target HIV-1 capsid via nonconventional MHC II restriction. Together, these findings indicate that TCR clonotypes with superior functions are associated with HIV control. Amplification or transfer of such clonotypes may contribute to immunotherapeutic approaches aiming at a functional HIV cure.
\end{abstract}

\section{Introduction}

HIV relentlessly destroys $\mathrm{CD}^{+} \mathrm{T}$ cells in the course of infection and causes functional impairment in the remaining $\mathrm{CD}^{+}$ $\mathrm{T}$ cell population. This leads to the progressive loss of adaptive responses to opportunistic pathogens and ultimately to the collapse of the immune system characteristic of AIDS. Chronic immune activation is thought to drive dysfunction of the remaining $\mathrm{CD}^{+} \mathrm{T}$ cell population, with both persistent viral antigenic stimulation and microbial translocation conspiring to exhaust $\mathrm{T}$ cell responses (1). Another parameter contributing to the loss of helper function may be the poor quality of $\mathrm{CD}^{+} \mathrm{T}$ cells that escape depletion. Early studies of the repertoire of $\mathrm{T}$ cell receptors (TCRs) documented a general loss of $\mathrm{CD} 4^{+} \mathrm{T}$ cell diversity in HIV-infected patients $(2,3)$, while further studies highlighted a preferential depletion of HIV-specific CD4 ${ }^{+} \mathrm{T}$ cells (4-6), suggesting that the HIV-specific repertoire was especially prone to diversity contraction. To date, the HIV-specific CD4 repertoire remains mostly uncharacterized at the molecular level, even

Authorship note: D. Benati and M. Galperin are co-first authors.

Conflict of interest: The authors have declared that no conflict of interest exists. Submitted: July 15, 2015; Accepted: March 8, 2016.

Reference information: J Clin Invest. 2016;126(6):2093-2108. doi:10.1172/JCI83792. though this information would be critical to define the potential for immune reconstitution in treated patients.

Rare cases of spontaneously controlled HIV-1 infection provide a unique opportunity to study the molecular characteristics of a fully functional $\mathrm{CD}^{+} \mathrm{T}$ cell response directed at HIV. Patients who maintain an undetectable viral load in standard assays ( $<50$ copies of HIV- $1 \mathrm{RNA} / \mathrm{ml}$ ) represent fewer than $0.5 \%$ of seropositive individuals but have a remarkably low risk of progressing to AIDS (7). These rare patients, called HIV controllers, or alternatively elite controllers, show signs of particularly efficient cellular responses that actively control the infected cell population (8). Controller CD8 ${ }^{+} \mathrm{T}$ cells have the capacity to potently inhibit HIV replication when added to cultures of infected autologous $\mathrm{CD} 4^{+} \mathrm{T}$ cells and are thought to play a key role in HIV control $(9,10)$. Recent evidence suggest that particular TCR clonotypes expressed by controller $\mathrm{CD} 8^{+} \mathrm{T}$ cells are responsible for their efficient cytotoxic responses, while HLA-matched non-controller patients show clonotypes of lower efficacy (11-13). In addition, clonotypes from controllers are able to maintain cross-recognition of dominant epitope variants, thus preventing the emergence of viral escape mutants $(11,12,14)$.

The role of the CD4 response in HIV control remains less completely understood. Controllers maintain a population of 
HIV-specific central memory $(\mathrm{CM}) \mathrm{CD} 4^{+} \mathrm{T}$ cells endowed with a strong proliferative capacity, which has been linked to autocrine IL-2 production and the upregulation of antiapoptotic molecules (15-18). However, the presence of long-lived CM cells does not appear sufficient to ensure HIV control, as patients treated early in the course of primary HIV infection also maintain specific $\mathrm{CM} \mathrm{CD} 4^{+} \mathrm{T}$ cells with strong proliferative capacity, but in most cases fail to control HIV replication upon treatment interruption (19). Multiple lines of evidence indicate that the antiviral CD4 response in controllers is qualitatively different from that in efficiently treated patients, and is not just a consequence of a very low viremia. In particular, controller $\mathrm{CD} 4^{+} \mathrm{T}$ cells preferentially target Gag rather than Env epitopes, suggesting differences in the repertoire of specific CD $4^{+} \mathrm{T}$ cells (20). Controller $\mathrm{CD} 4^{+} \mathrm{T}$ cells are also more polyfunctional, as indicated by the capacity to produce multiple cytokines and chemokines simultaneously (21). A key difference lies in the persistence of specific $C D 4^{+} \mathrm{T}$ cells with an effector differentiation status, even though the amount of HIV antigens available to drive these responses is minimal. We reported that Gag-specific $\mathrm{CD}^{+}{ }^{+} \mathrm{T}$ cells in controllers maintain a Th1 effector profile with IFN- $\gamma$ production and degranulation capacity, while such effectors disappear in patients treated in the long term (22). Controller CD4 ${ }^{+} \mathrm{T}$ cells express low levels of negative costimulatory molecules such as CTLA-4 and PD-1, compatible with preserved effector functions $(23,24)$. Taken together, these findings suggest that Th1 effectors escape depletion and retain optimal functions to sustain the antiviral response in controlled HIV infection.

An explanation for the remarkable properties of HIV-specific CD4 responses in controllers may lie in the nature of the TCRs that mediate these responses. We previously identified a population of $\mathrm{CD}^{+} \mathrm{T}$ cells with a high antigen sensitivity to immunoprevalent Gag peptides in HIV controllers, while this population was absent in treated patients (25). The sensitive detection of Gag antigens depended on intrinsic differences in the set of TCRs expressed by controller CD $4^{+} \mathrm{T}$ cells, as indicated by a high TCR avidity measured in MHC II tetramer dilution assays. High TCR avidity is a hallmark of viral control in several models of chronic viral infections (26) and has been associated with antiviral efficacy in HIV-specific CD $8^{+} \mathrm{T}$ cells $(10,13,27)$. In contrast, there are limited data available on the impact of TCR avidity on human $\mathrm{CD}^{+} \mathrm{T}$ cell antiviral responses. We reasoned that the presence of high-avidity TCRs may explain why controllers maintained HIV-specific $\mathrm{CD} 4^{+} \mathrm{T}$ cells with an effector differentiation status in spite of their very low viremia, and we set to characterize these TCRs at the molecular and functional level. The study was focused on TCRs specific for the most immunoprevalent CD4 epitope in Gag, located at position 293-312 in the capsid major homology region (MHR). This epitope, designated Gag293, is exceptionally immunoprevalent, as it induces an IFN- $\gamma$ ELISPOT response in close to half of HIV-1-infected patients irrespective of genetic background $(20,28)$ and gives a greater than $70 \%$ response rate in controllers of the Agence nationale de recherches sur le SIDA et les hépatites virales (ANRS) CO21 CODEX cohort (25). We could thus compare the repertoire of Gag293-specific clonotypes in patients of varied HLA backgrounds who controlled HIV infection either naturally or through antiretroviral therapy.

\section{Results}

We set out to compare Gag293-specific CD4 responses in HIV controllers and efficiently treated patients, two groups characterized by long-term viral control. The clinical and immunological characteristics of the studied patients are reported in Table 1. A stringent definition of HIV control was applied, based on an undetectable viral load in standard assays ( $<50$ copies HIV-1 RNA/ $\mathrm{ml}$ ) for more than 5 years. The duration of control was actually longer, as patients included in the controller group (HIC group, $n=14$ ) had been infected for a median duration of 19.6 years. They were compared with HIV-1-infected patients who had received long-term antiretroviral therapy (HAART group, $n=15$ ), with an undetectable viral load for at least 5 years and a median duration of treatment of 11.9 years. Thus, both groups were characterized by long-term viral suppression, which ensured that potential differences in CD4 responses were not primarily determined by levels of residual HIV viremia.

High antigen sensitivity of Gag293-specific CD4 responses in HIV controllers. TCR repertoire studies of specific $\mathrm{CD}^{+} \mathrm{T}$ cells have remained scarce in humans due to the limited clonal amplification of $\mathrm{CD}^{+} \mathrm{T}$ cells as compared with $\mathrm{CD} 8^{+} \mathrm{T}$ cells, and to the generally lower affinity of TCRs expressed by CD4 ${ }^{+} \mathrm{T}$ cells, which limits MHC II tetramer detection (29). In the case of HIV infection, these factors are compounded by the general decrease in the $\mathrm{CD}^{+} \mathrm{T}$ cell population and the preferential depletion of HIV-specific $\mathrm{CD}^{+} \mathrm{T}$ cells $(5,6)$. To address these issues, we devised a system of short-term primary $\mathrm{CD} 4^{+} \mathrm{T}$ cell line cultures that allowed the amplification of MHC II tetramer-positive cells prior to sorting. Using this system, we previously reported the presence Gag293-specific CD4 ${ }^{+} \mathrm{T}$ cells with high antigen sensitivity and high MHC II tetramer binding capacity in controllers, while such cells were absent in treated patients (25). To extend these results, we generated primary $\mathrm{CD} 4^{+} \mathrm{T}$ cell lines by stimulation with decreasing doses of Gag293 peptide. The specificity of $\mathrm{CD}^{+} \mathrm{T}$ cell lines was evaluated at equivalent growth stages (doubling time), by restimulation with Gag293 and analysis by IFN- $\gamma$ ELISPOT assay. As reported in Table 1, viable Gag293-specific cell lines were obtained for all patients of the HIC and HAART groups when initially stimulated with the highest peptide dose of $10^{-5} \mathrm{M}$. However, a marked difference was observed for cell lines generated at $10^{-7} \mathrm{M}$ peptide, with only 8 of 15 treated patients responding versus all of the 14 controllers $(P=0.006)$. The difference was also marked at the $10^{-9} \mathrm{M}$ peptide dose $(P=0.005)$. Moreover, two of the controllers, but none of the treated patients, responded at the $10^{-11} \mathrm{M}$ peptide dose. These experiments established that Gag293-specific CD4 ${ }^{+} \mathrm{T}$ cells had a higher antigen sensitivity in the controller group, as indicated by the capacity to proliferate and differentiate into IFN- $\gamma$-secreting effectors upon stimulation with minimal peptide doses.

Biased TRAV and TRBV gene usage in Gag293-specific CD4 ${ }^{+}$ $T$ cells. To characterize the Gag93-specific TCR repertoire, we first genotyped patients for the HLA-DRB1 gene. Eight controllers and 8 treated patients who shared at least 1 of $4 H L A-D R$ alleles (DR1, DR11, DR15, or DRB5) were included in the TCR study (Supplemental Table 1; supplemental material available online with this article; doi:10.1172/JCI83792DS1). The frequencies of these 4 alleles did not differ significantly between the HIC and the HAART groups 
Table 1. Clinical and immunological characteristics of patients

\begin{tabular}{|c|c|c|c|}
\hline Clinical parameters $^{A}$ & $\begin{array}{l}\text { HIV controllers } \\
\text { (HIC, } n=14)\end{array}$ & $\begin{array}{l}\text { Treated patients } \\
\text { (HAART, } n=15 \text { ) }\end{array}$ & $P$ value \\
\hline Age, yr & $50[34-61]$ & 48 [39-56] & $N S^{B}$ \\
\hline Duration of HIV-1 infection, yr & $19.6[9.8-26.0]$ & $11.9[6.8-25.3]$ & $0.015^{\mathrm{B}}$ \\
\hline Duration of antiretroviral treatment, yr & N/A & $11.1[5.6-19.3]$ & - \\
\hline Virus load, HIV-1 RNA copies/ml plasma & $<50$ & $<50$ & $N S^{B}$ \\
\hline $\mathrm{CD}^{+} \mathrm{T}$ cells $/ \mathrm{mm}^{3}$ & $875[648-1,400]$ & $570[266-1,534]$ & $0.008^{B}$ \\
\hline Nadir of $\mathrm{CD}^{+} \mathrm{T}$ cells $/ \mathrm{mm}^{3}$ & N/A & $216[21-589]$ & - \\
\hline \multicolumn{4}{|l|}{$\begin{array}{l}\text { Frequency of Cag293-specific CD4 }{ }^{+} T \text { cell } \\
\text { lines generated at different peptide doses }\end{array}$} \\
\hline Gag293 dose: $10^{-5} \mathrm{M}$ & $14 / 14$ & $15 / 15$ & $N S^{D}$ \\
\hline Gage 293 dose: $10^{-7} \mathrm{M}$ & $14 / 14$ & $8 / 15$ & $0.006^{0}$ \\
\hline Gag293 dose: $10^{-9} \mathrm{M}$ & $8 / 14$ & $1 / 15$ & $0.005^{0}$ \\
\hline Gag293 dose: $10^{-11} \mathrm{M}$ & $2 / 14$ & $0 / 15$ & $N S^{D}$ \\
\hline
\end{tabular}

( $P \geq 0.05$ by Fisher's exact test). CD $4^{+} \mathrm{T}$ cell lines from these patients were labeled with HLA-DR-matched Gag293-loaded tetramers and evaluated for the proportion of $\mathrm{Tet}^{+}$cells in the CD4 ${ }^{+} \mathrm{CD} 8$ $\mathrm{T}$ cell population (Figure 1A). Samples labeled with matched tetramers loaded with an irrelevant peptide (CLIP or Annexin II) were used as negative controls. The purity of the $\mathrm{Tet}^{+}$population was controlled after sorting, as shown in representative examples (Figure 1A, right). TCR diversity of the sorted cells was evaluated through CDR3 length polymorphism analysis, using the immunoscope technique $(30,31)$. TCR $\alpha$ variable gene (TRAV) expression in controller $\mathrm{Tet}^{+}$cells proved highly skewed, with a median of $44 \%$ of Gag293-specific cells expressing the TRAV24 gene family (Figure 1B), while this family was amplified at lower levels in specific cells of treated patients (median, $13 \% ; P=0.037$ ). In comparison, the TRAV24 family represented $1 \% \pm 0.2 \%$ of $\mathrm{T}$ cells in a control group of 7 healthy donors (data not shown). Analysis of CDR3 length distribution showed a Gaussian pattern for control peripheral blood mononuclear cells (PBMCs), as expected (Figure 1D). In contrast, Gag293-specific cells appeared oligoclonal, with a dominant peak corresponding to a CDR3 of 10 aa, according to the numbering scheme of the International ImMunoGeneTics Information System (IMGT) (32). This peak was more prominent in cell lines from controllers than those from treated patients, suggesting a stronger TCR bias in controlled HIV infection.

Analysis of TCR $\beta$ variable gene (TRBV) distribution also revealed a major bias in Gag293-specific cells, with a marked predominance of the TRBV2 family, expressed at a median value of $82 \%$ in the controller group (Figure 1C). TRBV2 was amplified in Tet $^{+}$cells of 7 of 8 controllers in the present study, while this amplification was seen in only 1 of 4 controllers in a first study, where we tested Gag293-specific TCR profiles with a panel of V $\beta$-specific antibodies (25). The lower frequency of TRBV2 (previously designated V 322 ) amplification detected in the initial study may have resulted from suboptimal $\mathrm{V} \beta$ antibody binding due to competition with the MHC II tetramer for binding to the TCR. The TRBV2 fam- ily also appeared amplified at high levels in some of the treated patient samples (median, 28\%), with a difference between the HIC and HAART groups that did not reach significance $(P=0.13$; Figure 1C). In comparison, $\mathrm{T}$ cells from healthy donors expressed $T R B V 2$ at low levels $(3.7 \% \pm 1.0 \%$; data not shown). CDR3 length distribution appeared more variable for the TRBV2 than the TRAV24 chain (Figure 1E), though a trend toward a predominant CDR3 of 13 aa was apparent in controllers' Tet $^{+}$ cells. Taken together, these data provide evidence for a highly skewed Gag293-specific TCR repertoire characterized by the preferential usage of the TRAV24 and TRBV2 variable gene segments. The fact that the bias is more marked in Gag293-specific cells from controllers than treated patients suggests a contribution of the TRAV24 and TRBV2 variable regions to the high-avidity recognition of Gag293.

High clonotypic diversity of Gag293-specific cells in controllers. Clonotypic repertoire analysis was carried out for the two variable gene families amplified in Gag293-specific cells, TRAV24 and TRBV2. PCR products corresponding to these two families were cloned, sequenced, and analyzed with IMGT tools (32). A minimum of 50 productive CDR3 sequences were analyzed for each sample (a full list of CDR3 sequences is provided in Supplemental Tables 2 and 3). Productive TRAV24 sequences (HIC: $n=584$; HAART: $n=496)$ were evaluated for diversity by counting the distinct clonotypes, i.e., the number of unique CDR3 aa sequences, present in each patient sample. The normalized number of clonotypes proved significantly higher in the HIC than the HAART group $(P=0.0011$; Figure 2A). Similarly, analysis of TRBV2 sequences (HIC: $n=716$; HAART: $n=566$ ) showed higher clonotypic diversity in HIC samples ( $P=0.0047$; Figure $2 \mathrm{~A})$. Of note, the number of TRBV2 clonotypes per 100 sequences was remarkably high in the HIC group (median, 36), indicating the presence of a diverse TCR repertoire in spite of the pronounced bias for TRBV2. Computation of Simpson's diversity index confirmed a trend for higher clonotypic diversity in the HIC group, which reached significance for TRAV24 but not TRBV2 sequences (Figure 2B). The number of mutations needed to generate the observed CDR3 sequences from their germline counterparts was also significantly higher in the HIC group, for both the TRAV24 and TRBV2 datasets (Supplemental Figure 4, A and B). Thus, both the number of clonotypes and the number of CDR3 insertions and deletions were consistent with the persistence of a more diverse Gag293-specific repertoire in controlled HIV infection.

Biased J and D gene usage in Gag293-specific TCRs from controllers. The Gag293-specific CDR3 sequence dataset was analyzed for the distribution of junction (J) and diversity (D) gene segments. TRAJ gene usage was restricted, with 15 and 11 distinct TRAJ genes detected in TRAV24 sequences for the HIC and HAART groups, respectively, of 61 TRAJ genes reported in the IMGT database (Figure 2C). The TRAJ17 gene predominated in the HIC group (48\%), while the next most abundant genes, TRAJ39 and TRAJ32, were present at $16 \%$ and $12 \%$, respectively. TRAJ gene usage in the HAART group appeared more evenly distributed among TRAJ17 
A DRB5

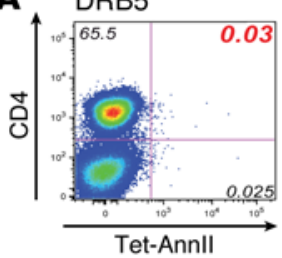

DR1

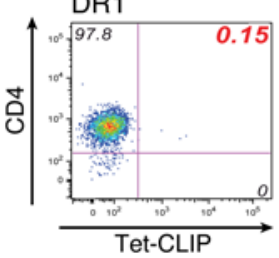

D

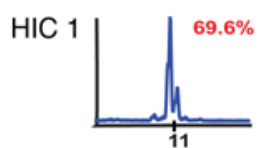

$\mathrm{HIC} 2$

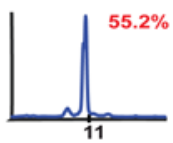

$\mathrm{HIC} 3$

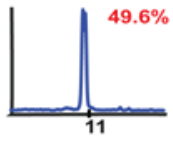

HIC 4

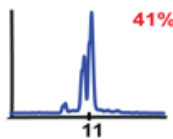

HIC 5

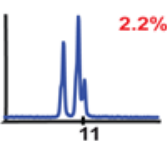

HIC 6

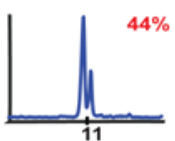

HIC 7

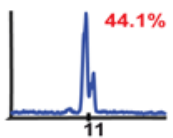

HIC 8
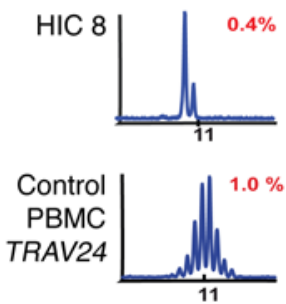
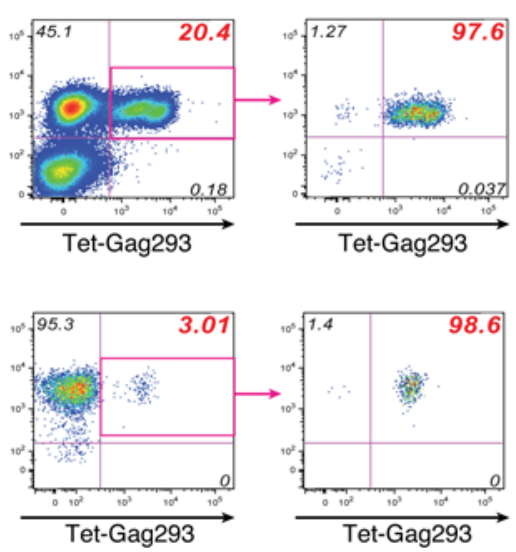

HAART 1

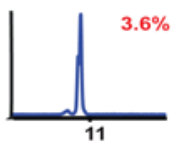

HAART

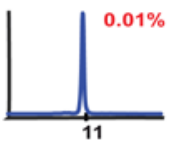

HAART 3

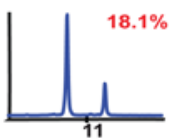

HAART 4

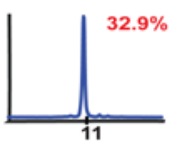

HAART 5

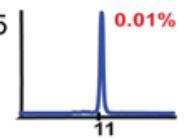

HAART

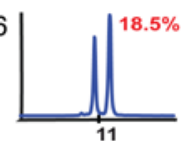

HAART 7

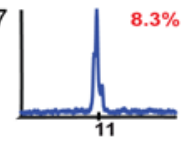

HAART 8

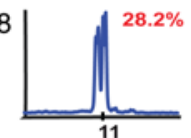

B

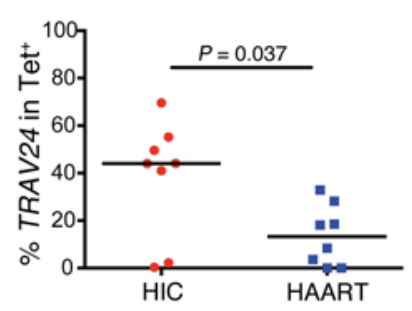

C

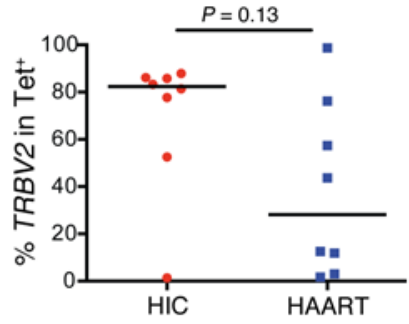

E

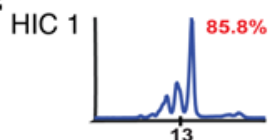

HIC 2

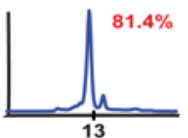

HIC 3

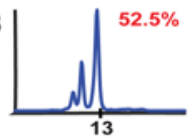

HIC 4

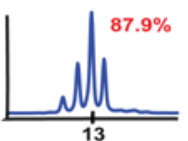

HIC 5

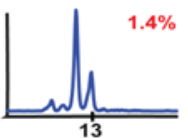

HIC 6

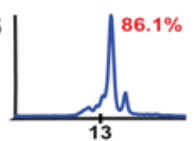

HIC 7

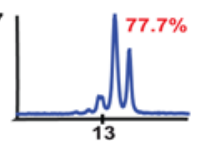

HIC 8
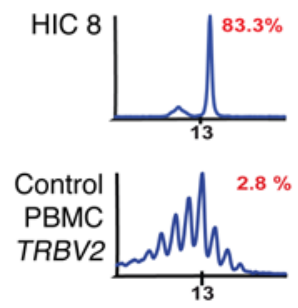

\begin{tabular}{l|l|l} 
HAART 1 & $3 \%$
\end{tabular}

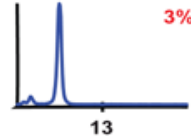

HAART 2

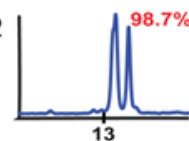

HAART 3

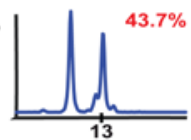

HAART 4

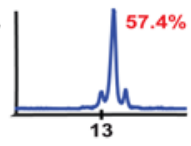

HAART 5

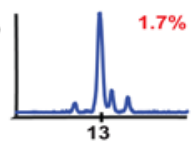

HAART 6

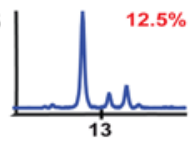

HAART 7

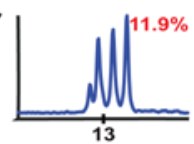

HAART 8
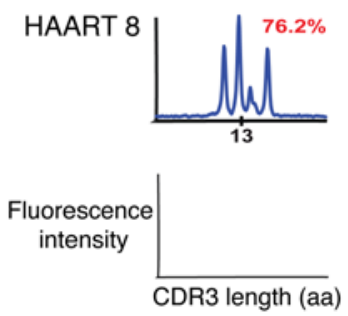

Figure 1. Immunoscope analysis of Gag293-specific CD4+ T cells. (A) Sorting of Gag293-specific CD4+ $\mathrm{T}$ cells with HLA-DR tetramers. Examples of primary $\mathrm{CD}^{+} \mathrm{T}$ cell lines labeled with DRB5 and DR1 tetramers. The percentage of Tet $^{+}$cells in the total CD4 ${ }^{+} \mathrm{T}$ cell population (middle plots) and in the sorted Tet ${ }^{+}$ population (right plots) is reported in red. Samples labeled with tetramers loaded with an irrelevant peptide (CLIP or Annexin II [AnnII]) were used as negative controls (left plots). (B and C) The percentages of Tet ${ }^{+}$cells expressing the TRAV24 (B) or TRBV2 family (C) were determined by qPCR in the HIC and the HAART groups and compared by using the Mann-Whitney $U$ test. (D and E) CDR3 length profiles for the TRAV24 (D) and TRBV2 families (E) are shown for each patient analyzed. Healthy donor PBMCs were used as controls (bottom left). The percentage of the TRAV24 or TRBV2 family in the total TRAV or TRBV PCR product is reported in red. (B-E) HIC group: $n=8$; HAART group: $n=8$. 
A

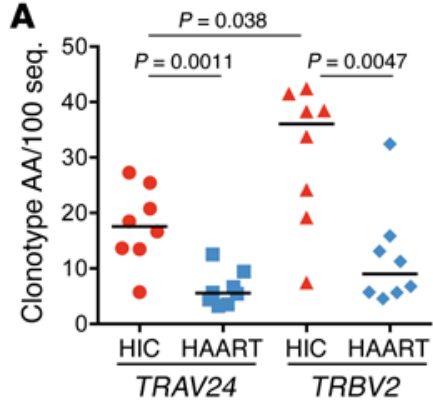

B

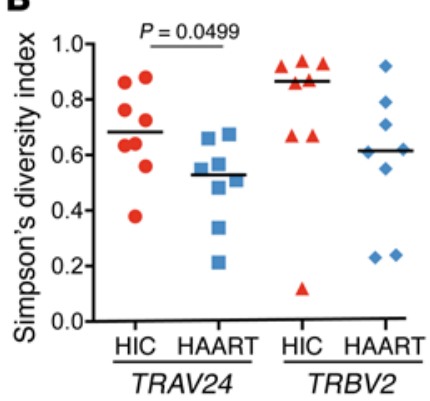

C

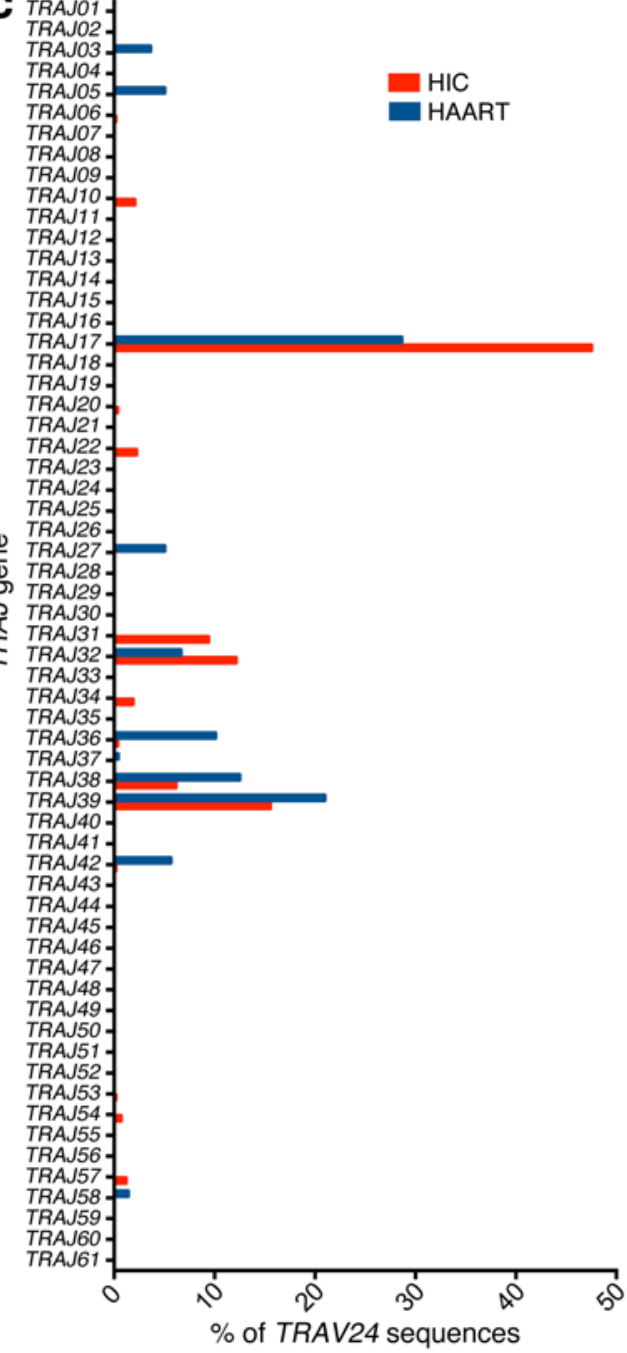

D

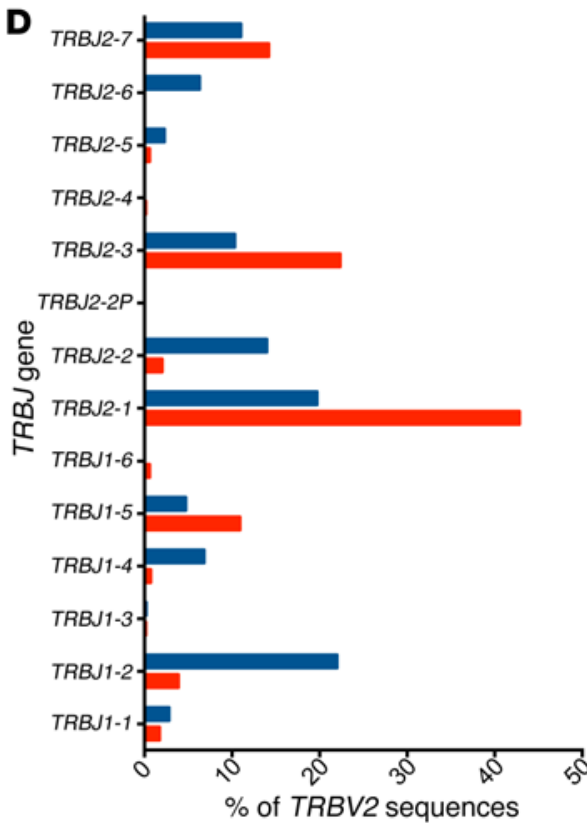

E

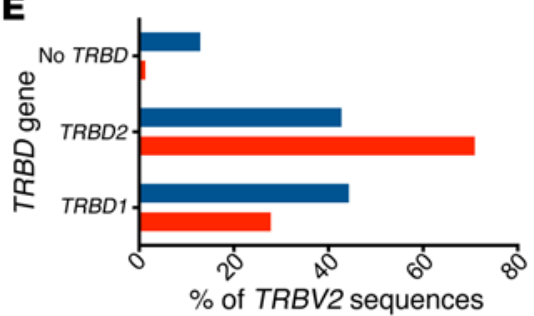

Figure 2. Clonotypic diversity of Gag293-specific TCRs. (A) The number of unique CDR3 amino acid sequences (clonotype AA) obtained per 100 TRAV24 or TRBV2 nucleotide sequences (seq.) was compared in the HIC and HAART groups with the Mann-Whitney $U$ test. (B) Simpson's diversity indexes computed for TRAV24 (left) and TRBV2 (right) clonotypes AA obtained in each patient were compared in the HIC and HAART groups with the Mann-Whitney $U$ test. (C-E) Frequencies of TRAJ genes (C), TRBJ genes (D), and TRBD genes (E) in Gag293-specific TRA or TRB sequences. Frequencies are reported for the HIC and HAART groups (red and dark blue bars, respectively). Number of sequences analyzed: HIC TRA): $n=584 ;$ HAART TRA): $n=496 ;$ HIC TRBJ and TRBD: $n=716 ;$ HAART TRBJ and TRBD: $n=566$.

(29\%), TRAJ39 (21\%), and TRAJ38 (13\%). Analysis of the TRBJ distribution in TRBV2 sequences showed again a more biased repertoire in the HIC group, with a predominant TRBJ2-1 gene present in $43 \%$ of sequences, while the most abundant TRBJ gene in the HAART group, TRBJ1-2, represented only $22 \%$ of sequences (Figure 2D). Of note, TRBJ1-2 was only minimally represented in HIC sequences (4\%), emphasizing differences between the HIC and HAART Gag293-specific repertoires. In addition, the TRBD2 gene segment was more prevalent in HIC sequences, while the two TRBD genes were equally represented in HAART sequences (Figure 2E). CDR3 lengths in the HIC group showed a marked peak at 10 and 13 aa for the TRAV24 and TRBV2 sequence sets, respectively, while CDR3 lengths were more evenly distributed in the HAART group (Supplemental Figure 4, C and D). These analyses indicated that Gag293-specific CDR3 sequences from controllers were biased in genetic composition, with a predominant use of TRAJ17, TRBJ2-1, and TRBD2, as well as narrowly distributed CDR3 lengths; in contrast sequences from treated patients appeared more heterogeneous. Thus, controller TCRs showed restricted V(D)J gene usage, indicative of a repertoire shaped under strong selective pressure, while maintaining a high clonotypic diversity.

High prevalence of public motifs in Gag293-specific TCRs from controllers. Given the observed biases in V(D)J gene usage, we next evaluated whether Gag293-specific CDR3 sequences shared common aa motifs. The MEME motif discovery software (http:// meme-suite.org/) was used in discriminative mode to identify motifs enriched in HIC as compared with HAART TRAV24 sequences. This approach revealed a highly prevalent but complex motif shared by $81 \%$ of HIC CDR3 sequences (Figure 3A). Comparison of the predominant motif in the HIC and HAART datasets highlighted that sequence differences mostly concentrated in the N-terminal part of the CDR3 (Supplemental Fig- 
A

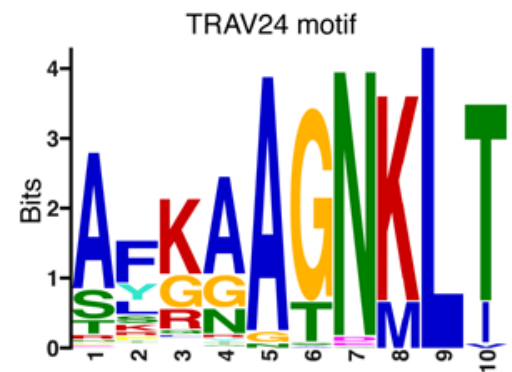

C
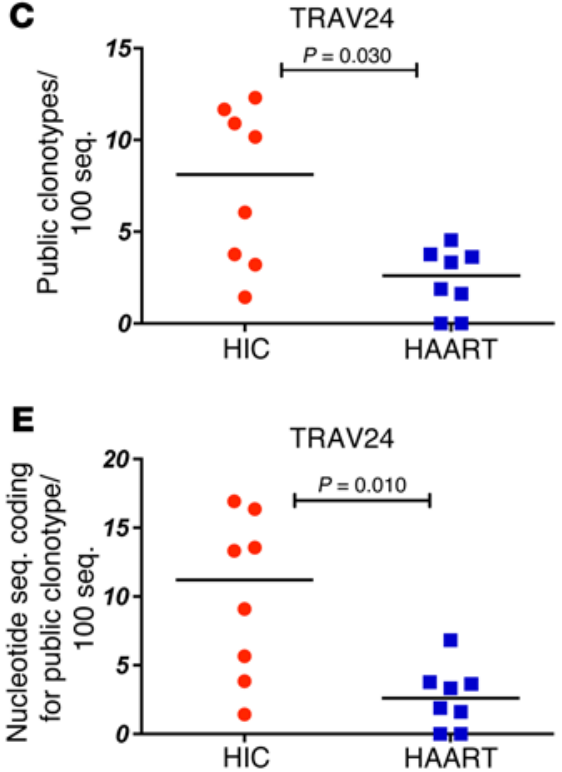

B

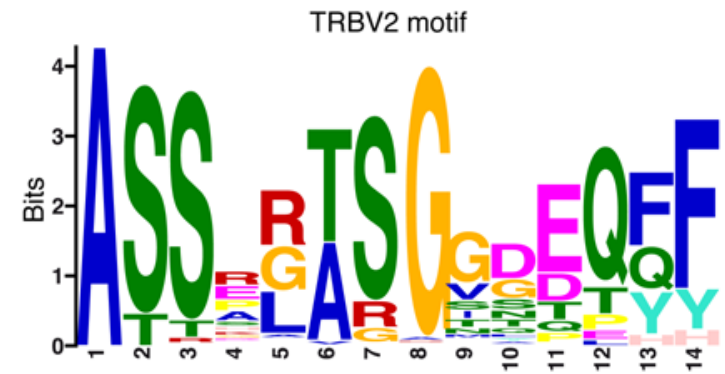

D
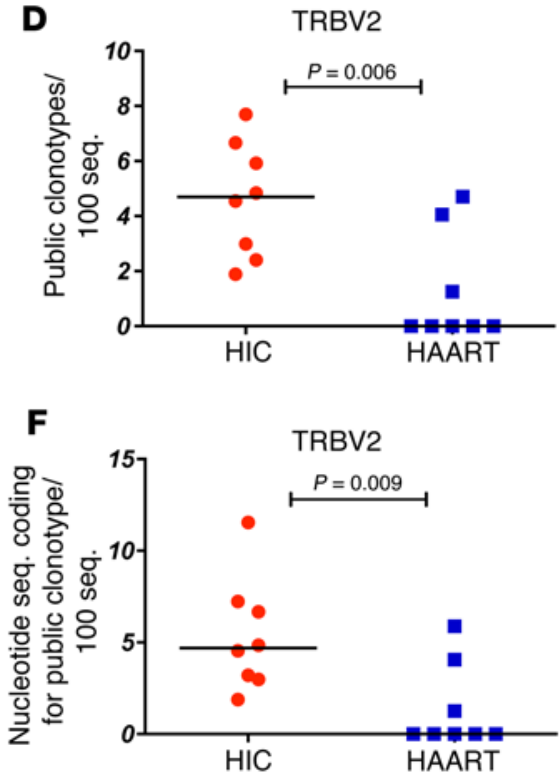

Figure 3. Quantification of public motifs and clonotypes in the Gag293-specific TCR repertoire. (A and B) The MEME motif discovery program was used to identify aa motifs enriched in controller TRAV24 sequences (A) and TRBV2 sequences (B) compared with corresponding sequences in treated patients. The MEME program was used in discriminative mode, which highlights differences between sequence datasets. The relative size of each aa symbol is proportional to its frequency in the HIC dataset, while the total height of aa symbols indicates the information content of the position in bits. (C and $\mathbf{D}$ ) Frequency of public clonotypes (clono.) per 100 TRAV24 sequences (C) or per 100 TRBV2 sequences (D) for each of the 8 controllers (HIC) and 8 treated patients (HAART) studied. (E and F) Frequency of nucleotide sequences coding for a public clonotype per 100 TRAV24 sequences (E) and per 100 TRBV2 sequences $(\mathbf{F})$. (C-F) Significant differences $(P<0.05)$ obtained by using the Mann-Whitney $U$ test are reported.

ure 5A). More detailed analyses identified two simpler motifs of 10 and 11 aa that were significantly more prevalent in HIC than HAART CDR3 sequences (Table 2). CDR3a motif AV24-1 ([A/S ] $\mathrm{x}[\mathrm{K} / \mathrm{R}]$ AAGNKLT; with $\mathrm{x}$ denoting any aa) encompassed the highly conserved AGNKLT sequence derived from the TRAJ17 gene, while motif AV24-2 (AxYGGATNKLI) contained a related ATNKLI sequence derived from the TRAJ32 gene. Taken together, these two motifs were found in $49 \%$ of HIC versus $29 \%$ of HAART TRAV24 sequences $(P<0.0001)$.

MEME analysis of TRBV2 sequences in discriminative mode identified a complex motif with interspersed highly conserved positions in $79 \%$ of HIC sequences (Figure 3B). A predominant motif was apparent in HIC TRBV2 sequences, while HAART $T R B V 2$ sequences appeared too diverse for motif identification (Supplemental Figure 5B). Further analyses of HIC sequences revealed the overlap of two simpler motifs of 13 and 14 aa, respectively (Table 2). CDR3 $\beta$ motifs BV2-1 (ASSx[R/G/L][T/A][S/G] Gxx $[\mathrm{E} / \mathrm{T}] \mathrm{Q}[\mathrm{F} / \mathrm{Y}])$ and BV2-2 (ASSx[R/G/L][T/A]SGGxx[E/T] $\mathrm{Q}[\mathrm{F} / \mathrm{Y}])$ were highly similar and differed only by the presence of an additional $G$ in the latter. Taken together, these two motifs were found in $50 \%$ of HIC versus $10 \%$ of HAART TRBV2 sequences $(P<0.0001)$, indicating a predominance of conserved CDR3 residues in HIC clonotypes. This bias suggested a requirement of particular CDR3 residues for efficient recognition of the Gag293MHC II complex. It was noteworthy that half of the TRAV24 and TRBV2 clonotypes identified in the HIC group shared the identified public motifs, indicative of a highly constrained Gag293-specific repertoire in controlled HIV infection.

High frequency of TCR sharing in HIV controllers. Public clonotypes were defined as identical CDR3 aa sequences found in at least two individuals, without any mismatch tolerated. A total of 18 public clonotypes were identified in the TRAV24 sequence dataset obtained from the 16 patients studied (Supplemental Figure $6 \mathrm{~A})$. The most prevalent public clonotype, AFKAAGNKLT (called TRAV24-F), was found in 6 controllers and 2 treated patients $(75 \%$ and $25 \%$, respectively), which are remarkably high frequencies in humans with diverse HLA II backgrounds. Groups of highly related public clonotypes were apparent, with a first group sharing 
Table 2. Prevalence of public motifs in Gag293-specific clonotypes

\begin{tabular}{|c|c|c|c|c|c|c|}
\hline & Motif sequence & $\begin{array}{l}\text { CDR3 length } \\
\text { (aa) }\end{array}$ & $\begin{array}{l}\text { HIC group } \\
\text { (\% seq.) }\end{array}$ & $\begin{array}{l}\text { HAART group } \\
\text { (\% seq.) }\end{array}$ & $\begin{array}{c}P \text { value, } \\
\text { HIC vs. HAART }\end{array}$ & $\begin{array}{l}\text { Public clonotypes } \\
\text { (\% seq) }\end{array}$ \\
\hline AV24-1 & {$[\mathrm{A} / \mathrm{S}] \times[\mathrm{K} / \mathrm{R}] \mathrm{AAGNKLT}$} & 10 & 37.16 & 22.38 & $<0.0001$ & 33.33 \\
\hline AV24-1 + AV24-2 & - & - & 48.80 & 29.03 & $<0.0001$ & 61.11 \\
\hline \multicolumn{7}{|l|}{ TRBV2 motif } \\
\hline BV2-1 & ASSx[R/G/L][T/A][S/G]Gxx[E/T]Q[F/Y] & 13 & 38.55 & 6.36 & $<0.0001$ & 50.00 \\
\hline
\end{tabular}

The percentage of motif occurrence is reported in total sequences from HIC and HAART patients as well as in the set of public clonotypes. Bold indicates the percentage of sequences carrying both motifs. $P$ values for differences between the HIC and HAART groups were computed with Fisher's exact test. $x$, any amino acid; seq., sequences.

the TRAJ17 chain and motif AxKAAGNKLT (AV24-1), and a second group sharing the TRAJ32 chain and motif AxYGGATNKLI (AV24-2). The 18 public clonotypes showed a high degree of motif sharing (61\% positive for motifs AV24-1 or AV24-2, Table 2), confirming their similarity. Of note, public clonotypes were more frequent in the HIC than in the HAART group $(P=0.03$; Figure $3 C)$. Counting the nucleotide sequences encoding public clonotypes, normalized to 100 sequences, confirmed the higher frequency of TCR sharing in the HIC group ( $P=0.01$; Figure $3 \mathrm{E})$.

Interestingly, the majority of TRAV24 public clonotypes (67\%) were encoded by multiple nucleotide sequences. The mean number of unique nucleotide sequences encoding a TRAV24 public clonotype was 2.33 , versus 1.05 for private (i.e., non-public) TRAV24 clonotypes. Detailed analysis of the 4 sequences coding for the most prevalent TRAV24 public clonotype, AFKAAGNKLT, showed that $\mathrm{P}$ and $\mathrm{N}$ mutations inserted between the TRAV24 and the TRAJ17 genes were few, ranging from 0 to 4 nucleotides (Supplemental Table 7). The numbers of nucleotides trimmed from germline sequences were also in the low range (6 to 10) compared with the ensemble of HIC sequences (mean of 9.9 trimmed nucleotides). The two predominant sequences, representing $64.9 \%$ and $33.8 \%$ of AFKAAGNKLT coding sequences, respectively, contained no mutation and were simply obtained by trimming 6 nucleotides from joined TRAV24-TRAJ17 genes. Thus, the most prevalent TRAV24 public clonotype could be easily generated from germline sequences, suggestive of convergent V(D)J recombination (33).

Public clonotypes were also abundant in Gag293-specific TCR $\beta$ chains, with a total of 18 found in the TRBV2 sequence dataset (Supplemental Figure 6B). Public clonotype sequences derived predominantly from the TRBJ2-3, TRBJ2-1, and TRBJ2-7 genes, which were all represented to a higher degree in HIC than HAART sequences. TRBV2 public clonotypes showed a remarkably high degree of motif sharing ( $83 \%$, Table 2), emphasizing the presence of conserved features in spite of relatively high sequence diversity. Again, TRBV2 public clonotypes were clearly more frequent in the HIC than the HAART group, in terms of aa sequences $(P=0.006$; Figure $3 \mathrm{D})$ and of nucleotide sequences ( $P=0.009$; Figure $3 F)$. The most frequent public clonotype, ASSRRTSGGTDTQY (called TRBV2-13), was found in 4 of 8 controllers (50\%) and absent in treated patients. The majority of TRBV2 public clonotypes (77\%) were encoded by more than one nucleotide sequence. The mean number of unique nucleotide sequences encoding a TRBV2 public clonotype was 2.28, versus 1.10 for private TRBV2 clonotypes, again suggesting that convergent $\mathrm{V}(\mathrm{D}) \mathrm{J}$ recombination contributes to the amplification of public clonotypes. Taken together, TRAV24 and TRBV2 sequence analyses revealed a highly biased Gag293-specific TCR repertoire, characterized by a high degree of TCR sharing in the controller group. TCR biases were of type III (identical CDR3 sequences) but also of type II (conserved CDR3 motifs) $(34,35)$, and characterized a substantial fraction of both TCR chains.

It was noteworthy that the majority of public clonotypes were restricted by multiple $H L A-D R$ alleles. Specifically, $83 \%$ of TRAV24 public clonotypes and 56\% of TRBV2 public clonotypes were identified in samples sorted with at least two different HLADR tetramers (Supplemental Figure 6, A and B). The most prevalent TRAV24 public clonotype, TRAV24-F, was restricted by the 4 HLA-DR alleles tested (DR1, DR11, DR15, and DRB5), while the most prevalent TRBV2 public clonotype, TRBV2-13, was restricted by 3 of these alleles (DR1, DR15, and DRB5). Thus, highly prevalent public clonotypes showed a high level of HLA cross-restriction, which could help explain their prevalence in patients of diverse genetic backgrounds. Of note, public clonotypes were also frequently restricted by two distinct $H L A-D R$ alleles within the same patient (Supplemental Tables 8 and 9), which could contribute to the induction of a more sensitive Gag293-specific response.

Ex vivo detection of Gag293-specific public clonotypes in HIV controllers. The Gag293-specific TCR repertoire was initially analyzed in primary $\mathrm{CD} 4^{+} \mathrm{T}$ cell lines, as the preferential depletion of specific $\mathrm{CD}^{+} \mathrm{T}$ cells in HIV-infected patients did not allow a direct ex vivo analysis of $\mathrm{Tet}^{+}$cells. However, the exceptional immunological status of HIV controllers made the ex vivo analysis feasible for a subset of these patients. Four samples sorted from controller PBMCs containing more than 2,000 $\mathrm{Tet}^{+}$cells were analyzed by immunoscope and sequencing. The DRB5 tetramer was used in these experiments, as it yielded the best sig$\mathrm{nal} /$ noise ratio (example in Figure 4A). Quantification of TRAV24 expression showed an amplification of this family in 3 of 4 HIC samples obtained ex vivo, as compared with the percentage of TRAV24 detected in PBMCs from 7 healthy donors $(1.0 \% \pm 0.2 \%)$ (Figure 4B). Quantification of TRBV2 showed an amplification in 


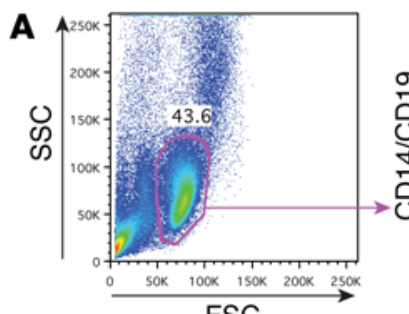

FSC

B

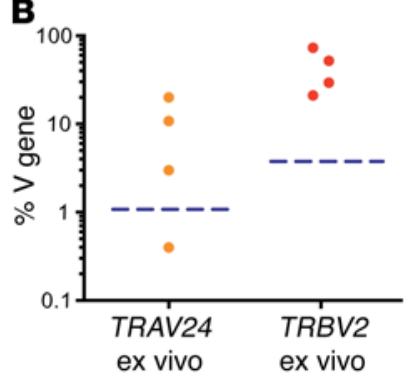

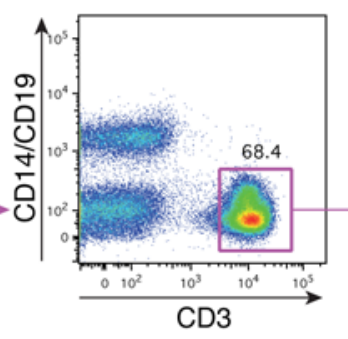

C

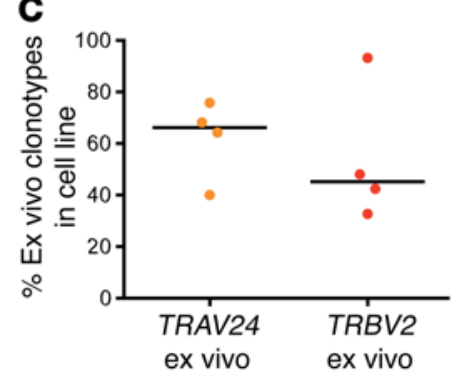

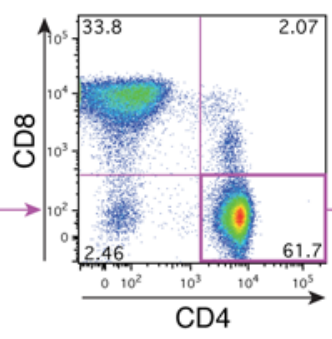

D

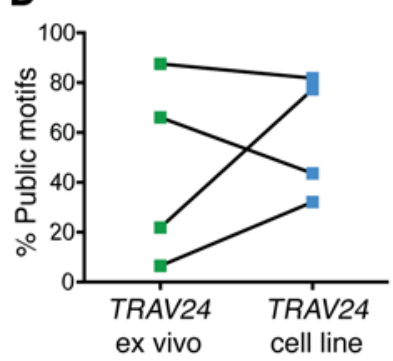

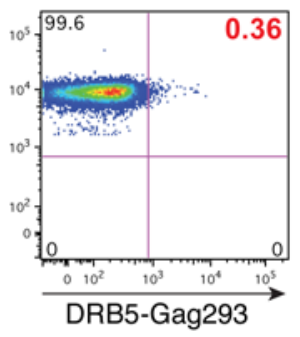

E

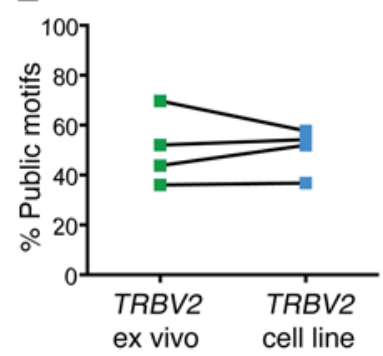

Figure 4. Ex vivo analysis of the Gag293-specific TCR repertoire. (A) Gating strategy for tetramer analysis in controller PBMCs. An example of PBMC staining with a control tetramer (AnnII) and a Gag293-loaded DRB5 tetramer is shown (plots at far right). One representative experiment of 4 is shown. (B) Frequency of TRAV24 and TRBV2 families in Gag293-Tet ${ }^{+}$cells sorted ex vivo. The percentages of TRAV24 expression in total TRAV products and TRBV2 expression in total TRBV products are reported. Dotted lines indicate the mean percentage of TRAV24 and TRBV2 families in CD4 ${ }^{+}$T cells from 7 healthy donors. (C) Representation of Gag293-specific clonotypes found ex vivo in the cell line obtained from the same patient. The percentage of sequences matching a TRAV24 or TRBV2 ex vivo clonotype in the corresponding cell line is reported, with medians indicated by horizontal lines. ( $\mathbf{D}$ and $\mathbf{E})$ The percentages of public motifs are compared in sequences obtained ex vivo and in the matched cell line for TRAV24 (D) and TRBV2 (E), using paired Student's t test.

4 of 4 HIC samples obtained ex vivo, as compared with PBMCs from healthy donors $(3.7 \pm 1.0 \%)$ (Figure $4 \mathrm{~B}$ ).

The frequency of each TRAV24 clonotype found ex vivo was computed relative to total TRAV24 sequences found in the same patient (Supplemental Table 10, third column) and in the matched cell line (fourth column). Ex vivo clonotypes represented a median of $66 \%$ of sequences found in matched cell lines, indicating a substantial overlap between the ex vivo and in vitro repertoires (Figure 4C). Similarly, the TRBV2 clonotypes detected ex vivo (Supplemental Table 3) represented a median of $45 \%$ of sequences found in matched cell lines (Figure 4C). Importantly, the frequency of TRAV24 public motifs (AV24-1 + AV24-2) did not differ significantly between ex vivo and cell line-derived sequences, and could be as high as $87 \%$ (Figure 4D). Frequencies of TRBV2 public motifs (BV2-1 + BV2-2) were also comparable ex vivo and in matched cell lines (Figure 4E). Public motifs were present at median values of $44 \%$ and $48 \%$ in the ex vivo TRAV 24 and TRBV2 sequence datasets, respectively, emphasizing the high level of motif sharing in the Gag293-specific repertoire.

Detailed analysis of TRAV24 sequences obtained ex vivo revealed the presence of previously identified public clonotypes in each of the patient tested (highlighted in color, Supplemental Table 10). Public clonotypes were present at median frequencies of $44 \%$ and $25 \%$ in ex vivo TRAV24 and TRBV2 sequences, respectively, which did not differ significantly from frequencies observed in matched cell lines (data not shown). Overall, the ex vivo analysis confirmed the high degree of TCR sharing among HIV controllers. It was striking that some of the most prevalent public clonotypes, such as AFKAAGNKLT, could represent more than half of TRAV24 sequences in the Gag293-specific repertoire (54.7\% in HIC7). This suggested that public clonotypes could shape the properties of the Gag-specific response in controlled HIV infection.

TCR transfer confers Gag293 recognition in the context of multiple HLA-DR alleles. We set out to functionally characterize the most prevalent TRAV24 public clonotype, AFKAAGNKLT (clonotype TRAV24-F), by pairing it with different TRBV2 chains and testing the activity of reconstituted TCRs. The chosen TRBV2 clonotypes, no. 24 (CASSRLAGGMDEQFF) and no. 25 (CATTPGASGISEQFF), were the most abundant in the cell line of patient HIC2 $(10.38 \%$ and $33.96 \%$ of TRBV2 chains, respectively; Supplemental Table 3), and were coexpressed at high levels with clonotype TRAV24-F, resulting in a high probability of functional TCR chain pairing. The third TRBV2 clonotype, no. 5 (CASSGLAGGMDEQFF), was derived from patient HIC3, who also expressed TRAV24-F at a high level. Clonotype 5 differed by a single residue ( $R$ to $\mathrm{G}$ ) from clonotype 24 and thus provided information on the contribution of the CDR3 $\beta$ arginine to TCR function. TRBV2 clonotypes 24 and 5 carried the public motif BV2-1, while TRBV2 clonotype 25 was private (see Supplemental Table 11 for a list of all TCR constructs). Fulllength TRA and TRB genes were cloned into a T2A-containing lentiviral vector, ensuring equimolar expression of the two chains. The resulting TCRs, F24, F25, and F5, were transduced in J76, a mutant Jurkat cell line defective for endogenous TCR expression, which provides a favorable cellular context for TCR transfer assays. After transduction, J76 cells expressed equivalent levels of the 3 TCRs at the cell surface ( $\geq 60 \% \mathrm{TCR}^{+}$cells) and recovered a high level of CD3 surface expression (Figure $5 \mathrm{~A}$ ).

To test the specificity of the transferred TCRs, we labeled transduced J76 cells with a panel of Gag293-loaded HLA-DR tetramers. The F24 TCR was found to bind 3 of the 4 tetramers 
A
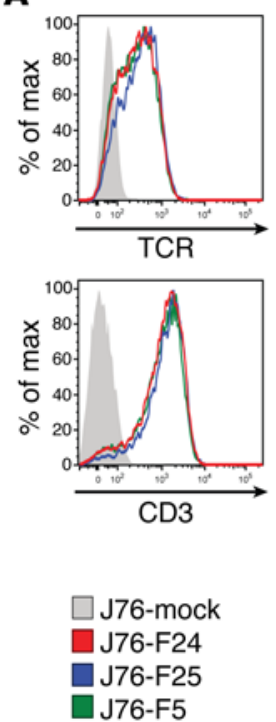

$\square$ J76-mock

J76-F25

$\square$ J76-F5
B Control J76

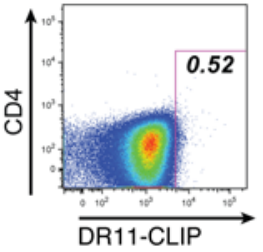

J76-F24

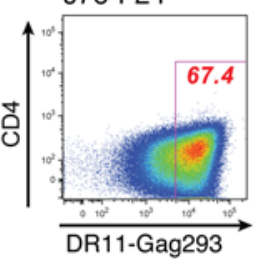

C

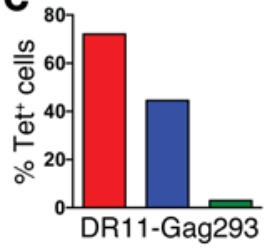

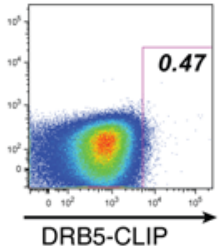
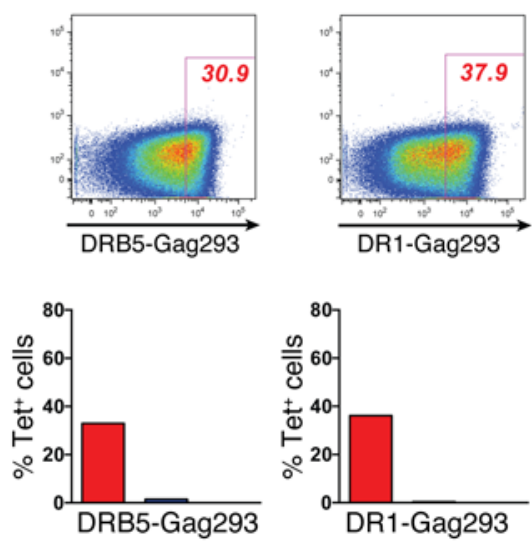
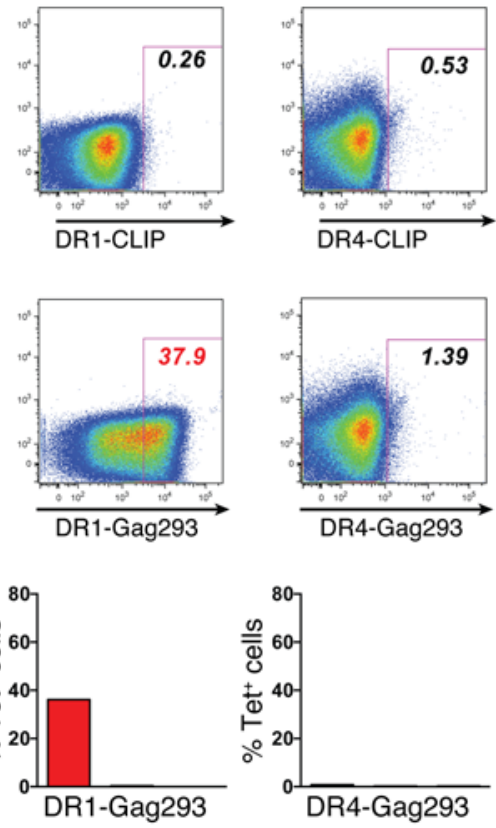

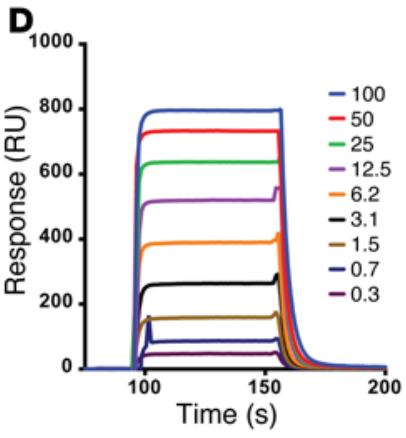

DR1-Gag293

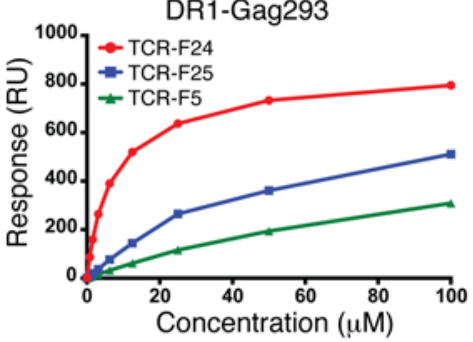

E
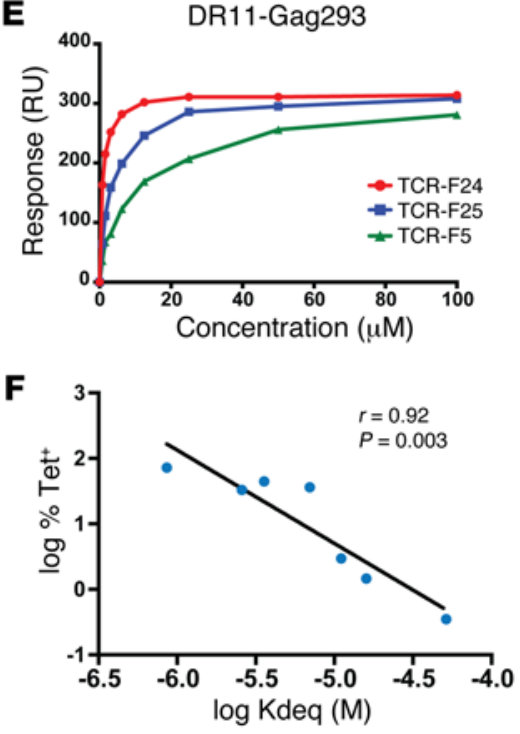

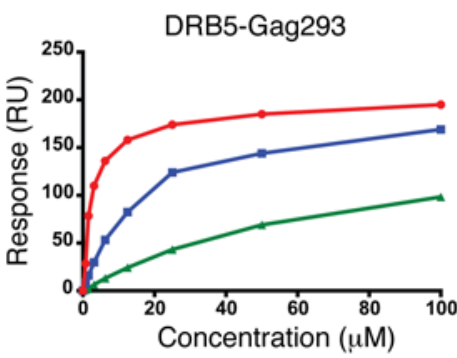

Figure 5. Public TCRs confer MHC II cross-restriction and high-affinity Gag293-MHC binding. (A) Expression of TCR $\beta$ and CD3 in 176 cells transduced with the F24, F25, and F5 TCRs. Solid gray histograms correspond to mock-transduced J76 cells. (B) Staining of F24-transduced J76 cells with CLIP-loaded tetramers and Gag293-loaded tetramers. The percentage of Tet $^{+}$cells is reported in each plot. One representative experiment of 4 is shown. (C) The percentage of Tet ${ }^{+}$cells after transfer of F24, F25, and F5 is reported for each of the 4 tetramers tested after subtraction of CLIP-tetramer background (mean of 2 experiments). (D) Example of SPR sensorgrams. The soluble F24 TCR (concentrations 0.3 to $100 \mu \mathrm{M}$ ) was flown over immobilized DR11-Gag293 monomers to measure the SPR response. RU, response units. (E) Affinity measurement of the F24, F25, and F5 TCRs for Gag293 complexed to DR11, DRB5, or DR1 monomers. Each soluble TCR was flown over Gag293-DR complexes at different concentrations ( $x$ axis) to measure binding RU ( $y$ axis). Representative of $n>2$ experiments. (F) Correlation between TCR affinity and tetramer binding. TCR affinities (log Kdeq) of the F24, F25, and F5 TCRs for the 3 Cag293DR complexes are plotted in function of the percentage of Gag293-Tet ${ }^{+}$cells (log \% Tet ${ }^{+}$) for the corresponding TCR/HLA-DR combination ( $y$ axis). $r$, linear regression coefficient. $P$ value: probability that the slope estimated by linear regression is significantly non-zero.

tested, with an efficiency that was higher for DR11 as compared with DRB5 and DR1 (Figure 5B). Quantification of tetramer binding (Figure 5C) showed that the 3 TCRs recognized Gag293 in the context of DR11, with a labeling that was strong for F24 (72\% $\left.\mathrm{Tet}^{+}\right)$, intermediate for F25 $\left(44 \% \mathrm{Tet}^{+}\right)$, and low for F5 $\left(3 \% \mathrm{Tet}^{+}\right)$. F24 bound the DRB5 tetramer at intermediate levels $\left(33 \% \mathrm{Tet}^{+}\right)$, while binding was weak for F25 (1.5\%) and undetectable for F5. Binding with the DR1 tetramer showed a similar pattern. The DR4 tetramer provided a negative control, as it did not bind any of the TCRs tested. These experiments indicated that the highly prevalent public clonotype TRAV24-F could confer Gag293 recognition in the context of multiple HLA-DR molecules, with an efficiency 
Table 3. TCR affinities determined by SPR

$\begin{array}{lc}\text { TCRs } & \begin{array}{c}\text { DRB1*1101 Kdeq } \\ (\mu \mathrm{M})\end{array} \\ \text { F24 } & 0.86 \pm 0.15 \\ \text { F25 } & 3.58 \pm 0.25 \\ \text { F5 } & 11.06 \pm 1.73\end{array}$

DRB1*0101 Kdeq $^{*}(\mu \mathrm{M})$
$6.97 \pm 0.22$
$51.50 \pm 3.00$
$>100$

Affinities (Kdeq) of the F24, F25, and F5 TCRs for Gag293-loaded HLA-DRB monomers. Each Kdeq value represents the mean \pm SEM from at least 2 independent experiments performed in duplicate.

that depended both on the presenting $H L A-D R$ allele and on the nature of the TRBV2 chain.

Tetramer titration experiments showed the expected hierarchy among the 3 TCRs (F24>F25>F5) but did not prove sensitive enough to determine TCR avidity for F25 and F5 (Supplemental Figure 12). To precisely determine TCR affinity, we expressed the F24, F25, and F5 TCRs as soluble recombinant proteins and measured their binding to Gag293-HLA-DR monomers by surface plasmon resonance (SPR). The soluble TCRs were passed over chips coated with immobilized DR11, DRB5, or DR1 monomers loaded with Gag293 (example of sensorgram in Figure 5D). In agreement with tetramer binding assays, the 3 TCRs recognized Gag293-HLA-DR complexes with different affinities (F24>F25>F5). This hierarchy was conserved across the different HLA-DR alleles tested (Figure 5E). Of note, the F24 TCR recognized the Gag293-DR11 complex with a very high affinity, of $0.86 \mu \mathrm{M}$ (Table 3), which is rarely seen for MHC II-restricted TCRs (36). F24 bound the Gag293-DRB5 and Gag293-DR1 complexes with affinities that were 3- and 8-fold lower, respectively, but still remained in the high-affinity range $(<10 \mu \mathrm{M})$. The F25 and F5 TCRs also interacted with the Gag293-DR11 complex with relatively high affinities ( 3 and $11 \mu \mathrm{M}$, respectively), while affinities were intermediate or low for the two other allomorphs tested. The percentage of Tet $^{+}$cells in TCR-transduced J76 cells, when measurable, correlated well with affinities measured by SPR ( $r=$ 0.92; Figure 5F), consistent with the notion that tetramer binding predominantly depends on TCR affinity. These results showed that association of the TRAV24-F public clonotype with highly expressed TRBV2 clonotypes could, for certain combinations, generate HLA-cross-restricted TCRs of unusually high affinity.

Transfer of TCRs containing public TRAV24 and TRBV2 clonotypes confers high antigen sensitivity to $\mathrm{J} 76 \mathrm{cells}$. TCR function was tested by monitoring the induction of the early activation marker CD69 in transduced J76 cells. Murine fibroblastic L cells engineered to express a single human $H L A-D R$ allele were used as antigen-presenting cells (APCs), allowing precise control of the restricting HLA molecule. L cells expressing DR1, DR11, DR15, or DRB5 (Figure 6A) and DR3, DR4, or DR7 (Supplemental Table 11) were pulsed with decreasing doses of Gag293 peptide and cocultured with J76 cells transduced with the F24, F25, and F5 TCRs. The 3 TCRs could signal and trigger T cell activation, as indicated by the induction of CD 69 in $\geq 50 \%$ of J76 cells in the presence of DR11 APCs. The F24 TCR showed the broadest reactivity, as it induced strong $\mathrm{T}$ cell activation in the presence of DR11, DR15, DRB5, and DR1 APCs, and weak but consistent T cell activation in the pres- ence of DR7 APCs (Supplemental Table 11). When restricted by DR11, F24 antigen sensitivity reached $4 \times 10^{-7} \mathrm{M}$ as measured by the half maximal effective concentration $\left(\mathrm{EC}_{50}\right)$ for $\mathrm{CD} 69$ induction, which represents a high sensitivity for a cell culture system devoid of costimulatory molecules. The F25 TCR did not react with DR7 and showed intermediate reactivity with the other $4 \mathrm{HLA}$ $D R$ alleles. The F5 TCR showed the narrowest restriction, with an antigen sensitivity of $2 \times 10^{-6} \mathrm{M}$ in the presence of DR11 APCs, low responses in the presence of DR15 and DRB5 APCs, and undetectable responses in other cases. TCR affinities measured by SPR for the $D R 11, D R B 5$, and $D R 1$ alleles correlated well with antigen sensitivities measured in transduced J76 ( $r=0.85, P=0.016$; Figure 6D), suggesting that TCR affinity dictated TCR function.

Two other TRAV24 public clonotypes, TRAV24-S (ASKAAGNKLT) and TRAV24-RR (SRRAAGNKLT), conferred cross-restriction by $4 H L A-D R$ alleles when paired to TRBV2 chains 24 and 5, respectively (Supplemental Table 11), emphasizing that broad HLA cross-restriction was a frequent property of public clonotypes. We then tested the pairing of TRAV24-F with two TRBV2 public clonotypes: TRBV2-4 (ASSPGTSGVGEQF), shared by 2 controllers; and TRBV2-13 (ASSRRTSGGTDTQY), the most prevalent TRBV2 clonotype, shared by 4 controllers. The resulting TCRs, F4 and F13, were cross-restricted by $3 H L A-D R$ alleles (DR11, DR15, and DRB5) (Figure 6B). While F4 had relatively good antigen sensitivity $\left(10^{-6} \mathrm{M}\right)$ when restricted by DR11, it gave only low responses with the two other alleles. In contrast, F13 showed good antigen sensitivity with DR11 $\left(7.5 \times 10^{-7} \mathrm{M}\right)$ and intermediate sensitivity with the two other alleles (Supplemental Table 11). F13, like F24, carried an R at position 5 of the TRBV2 CDR3 junction, which may contribute to the efficient detection of Gag293 in varied HLA backgrounds. Two TCRs derived from private clonotypes from treated patients were studied for comparison. TCRs HD5 and HY9 comprised the most prevalent TRAV24 and TRBV2 clonotypes from treated patients HAART3 and HAART6, respectively (Supplemental Table 11). These TCRs yielded responses of medium avidity when restricted by DR15 and DR1 $\left(10^{-5} \mathrm{M} \leq \mathrm{EC}_{50}\right.$ $\leq 10^{-6} \mathrm{M}$ ), gave only low responses in the presence of DRB5 L cells, and did not react with DR11 L cells (Figure 6C). While these TCRs from treated patients could react with several HLA-DR alleles, their degree of cross-restriction appeared narrower than that observed for controller TCRs. Taken together, the analysis of recombinant TCRs specific for Gag293 highlighted that the most prevalent public clonotypes conferred efficient Gag293 recognition in the context of multiple $H L A-D R$ alleles. Interestingly, antigen sensitivity measured for the 8 TCRs tested in the presence of DR11 correlated with the number of $H L A-D R$ alleles restricting each of these TCRs $(r=-0.86, P=0.006$; Figure 6E), suggesting that both properties depended on the same TCR features.

We next verified that the recombinant TCRs could recognize native HIV capsid antigens naturally processed by APCs, in addition to peptide-pulsed APCs. To this end, we used infected monocyte-derived dendritic cells (MDDCs) as alternative APCs. MDDCs from DR11 ${ }^{+}$healthy donors were infected with an HIV-1 pseudotyped virus and incubated with J76 cells expressing the F24, F25, or F5 TCR. The TCR-transduced cells showed robust CD69 induction in the presence of infected MDDCs, while CD69 expression remained moderate in the absence of infection (Figure 6F). CD69 
A

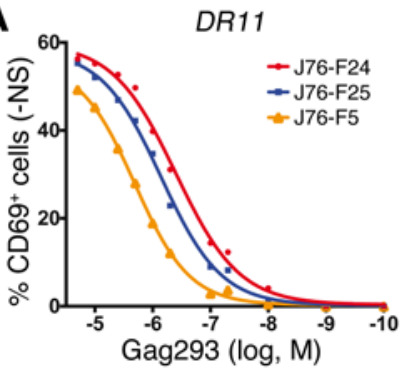

B
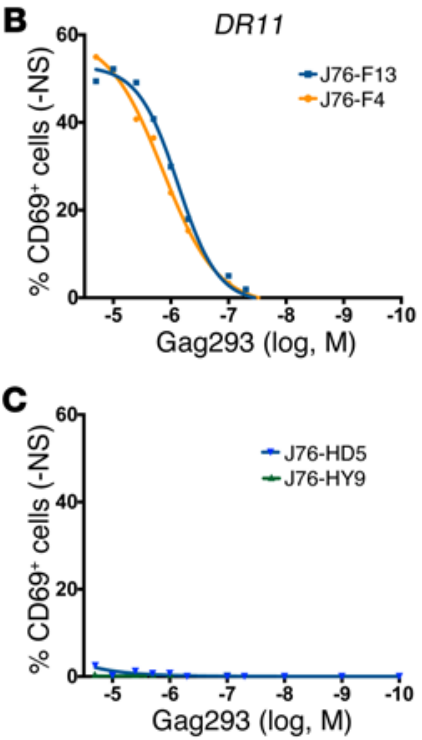

D

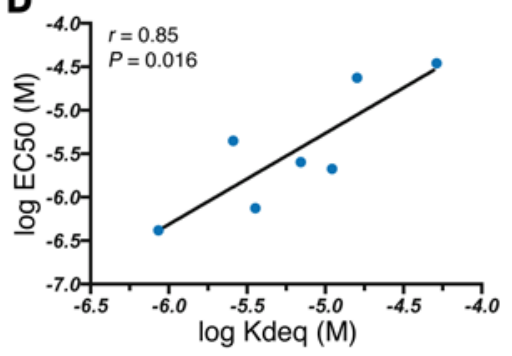

DR15

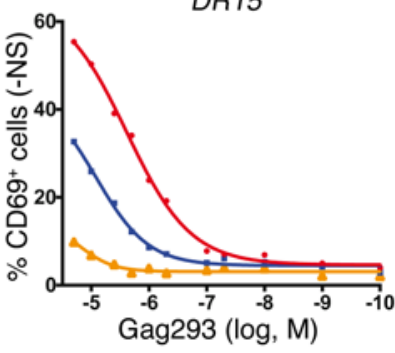

DR15
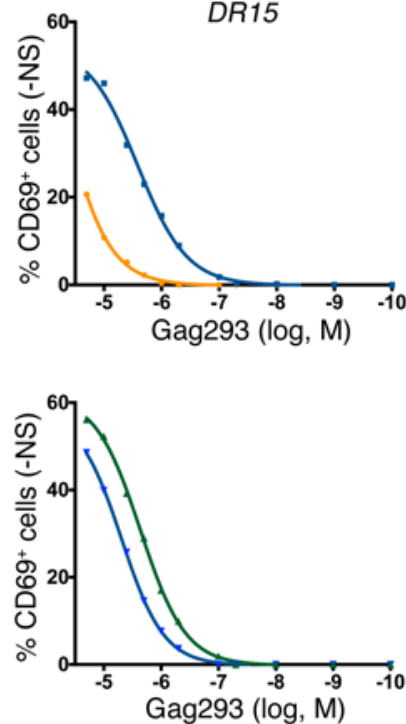

DRB5
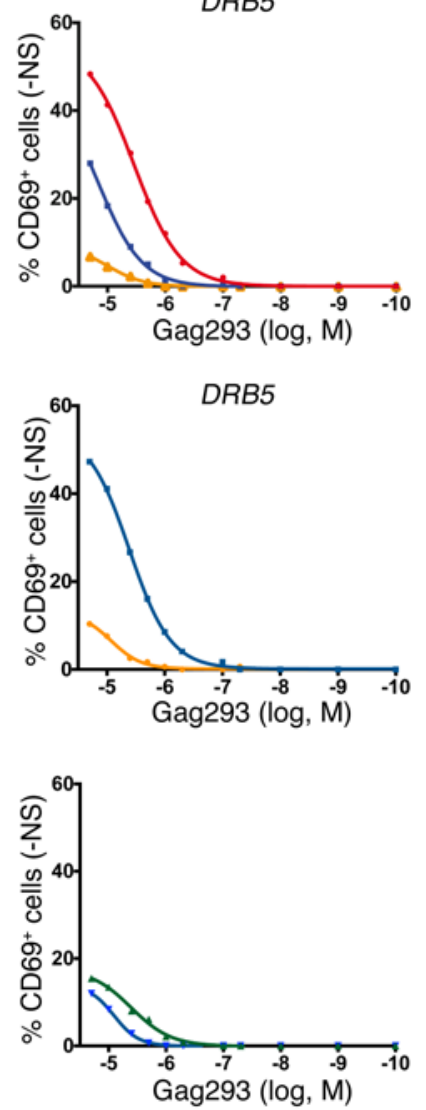

DR1

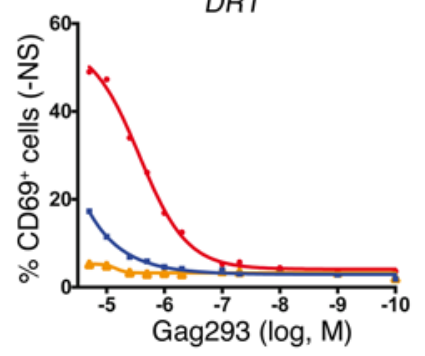

$D R 1$
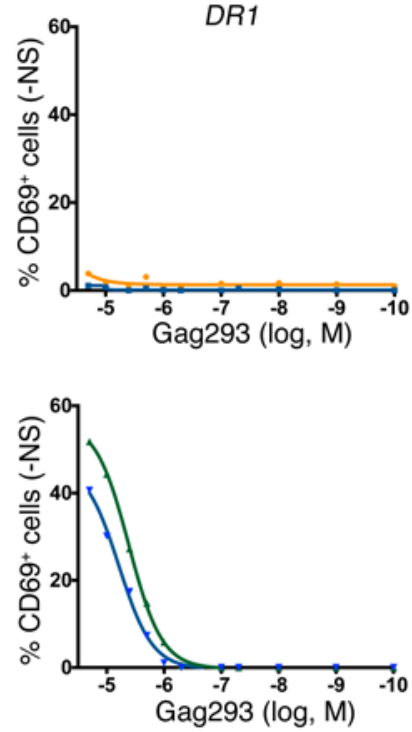
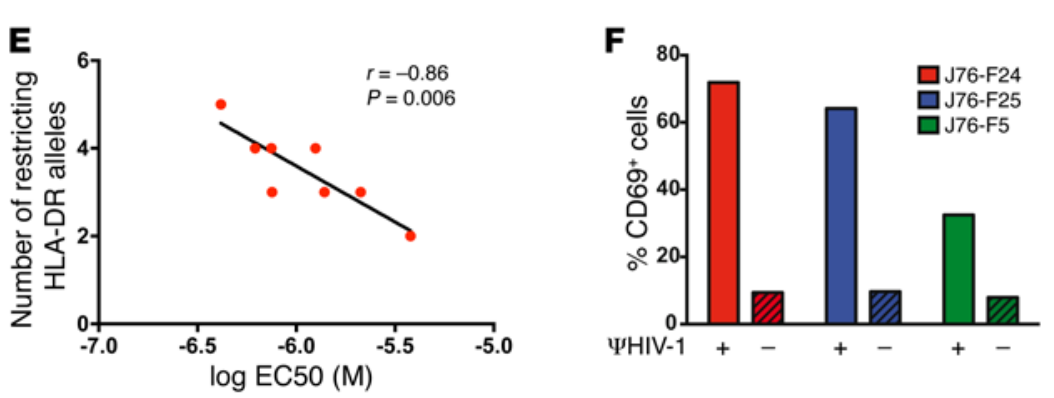

Figure 6. Public TCR transfer confers high antigen sensitivity to $\mathbf{7 6}$ cells. (A and B) Antigen sensitivity assay in TCR-transduced J76 cells. Percentages of CD69 expression in 776 cells transduced with TCRs F24, F25, or F5 (A) or with F4 or F13 (B), after coculture with L cells expressing different $H L A-D R$ alleles (DR11, DR15, DRB5, or DR11) and loaded with decreasing Cag293 concentrations. (C) Antigen sensitivity assay of TCRs HD5 and HY9 from HAART patients. -NS, not stimulated. (A-C) Experiments were conducted in triplicate, with curves corresponding to one experiment shown for clarity. (D) Correlation

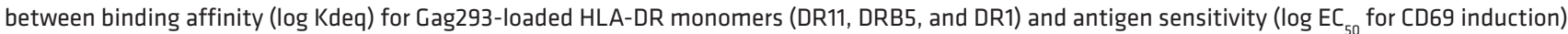
of the F24, F25, and F5 TCRs. (E) Correlation between the antigen sensitivity ( $\log$ EC $_{50}$ ) measured for 8 HIC TCRs (described in Supplemental Table 11) in the presence of DR11 APCs and the number of HLA-DR alleles restricting these TCRs. (D and E) $r$, linear regression coefficient; $P$ value: probability that the slope estimated by linear regression is significantly non-zero. (F) TCR reactivity to native HIV-1 capsid antigens. CD69 induction was quantified in TCR-transduced $J 6$ cells cocultured with dendritic cells infected with the VSV-pseudotyped virus $\Psi$ HIV-1 (solid bars) or left uninfected (striped bars). One representative experiment of 3 is shown.

induction followed the same hierarchy as that observed when $\mathrm{L}$ cells were used as APCs (F24>F25>F5), indicating that TCR properties were conserved in different antigen presentation systems.

As the public clonotypes consistently generated TCRs with optimal antigen sensitivity in the context of DR11, it was important to verify that differences in antigen sensitivity between the HIC and the HAART group did not depend on a bias in DR11 expression. Among the 29 patients included in the study, 3 controllers and 4 treated patients expressed a DR11 allele, including HLA-
DRB1*1101, -DRB1*1122, and -DRB1*1165 (Supplemental Table 1). Comparison of the last Gag293 peptide dilution that induced a specific cell line showed that, among DR11 patients, 3 of 3 controllers and 1 of 4 treated patient responded at a dilution less than $10^{-5} \mathrm{M}$ (Supplemental Figure 13A). Trends for a higher Gag293-specific ELISPOT response and for more prominent TRAV24 biases were also noted in DR11 controllers as compared with DR11-treated patients (Supplemental Figure 13, B and C). Taken together, these findings support the notion that particular clonotypes expressed 
A
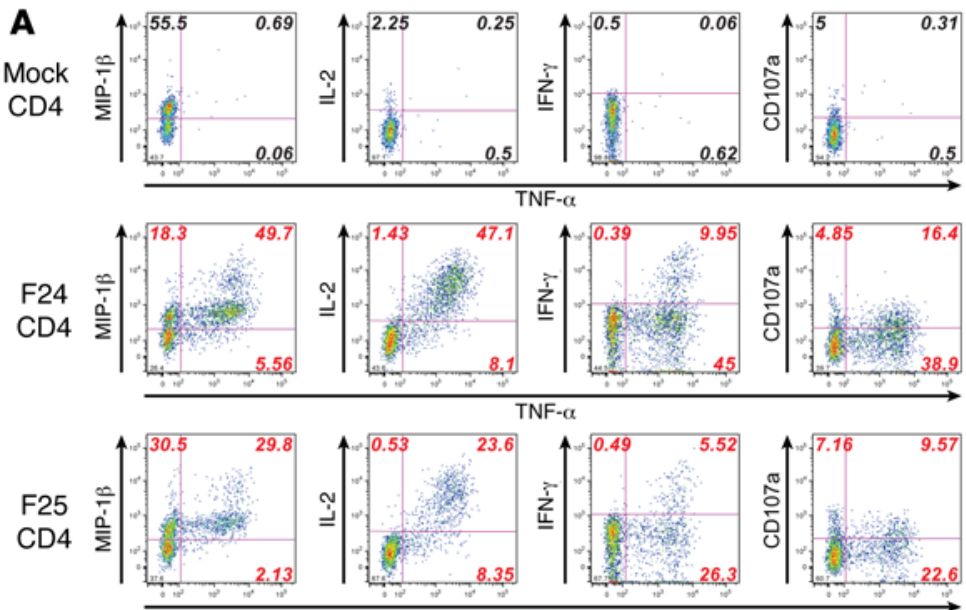

TNF- $\alpha$
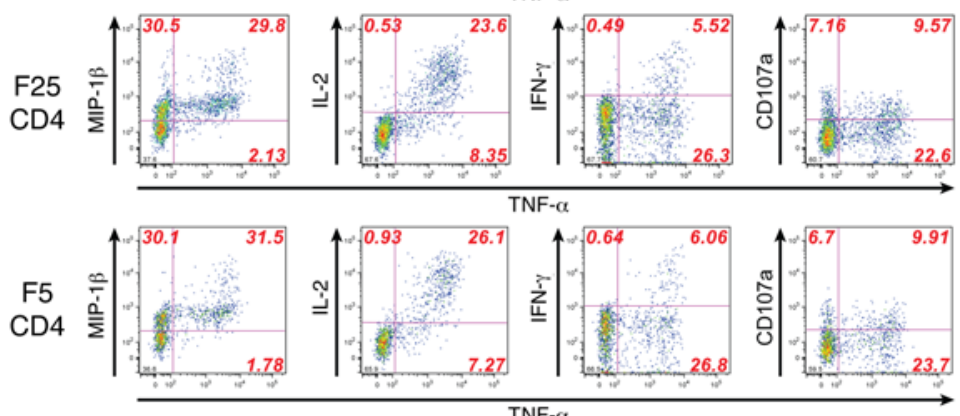

TNF- $\alpha$
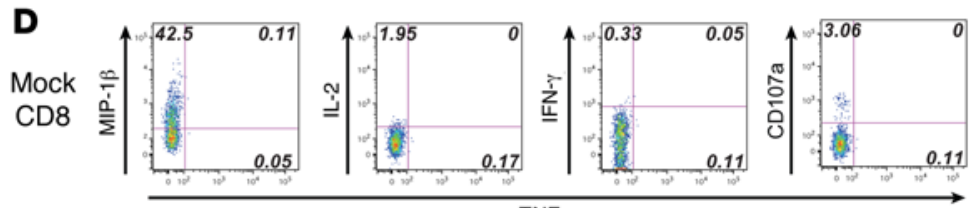

TNF- $\alpha$
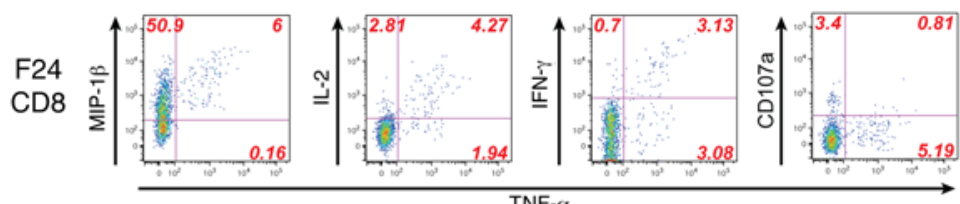

TNF- $\mathrm{C}$
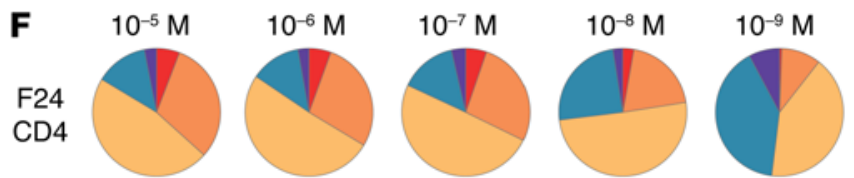

PMA/iono

F25
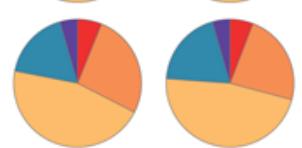

F5

CD4
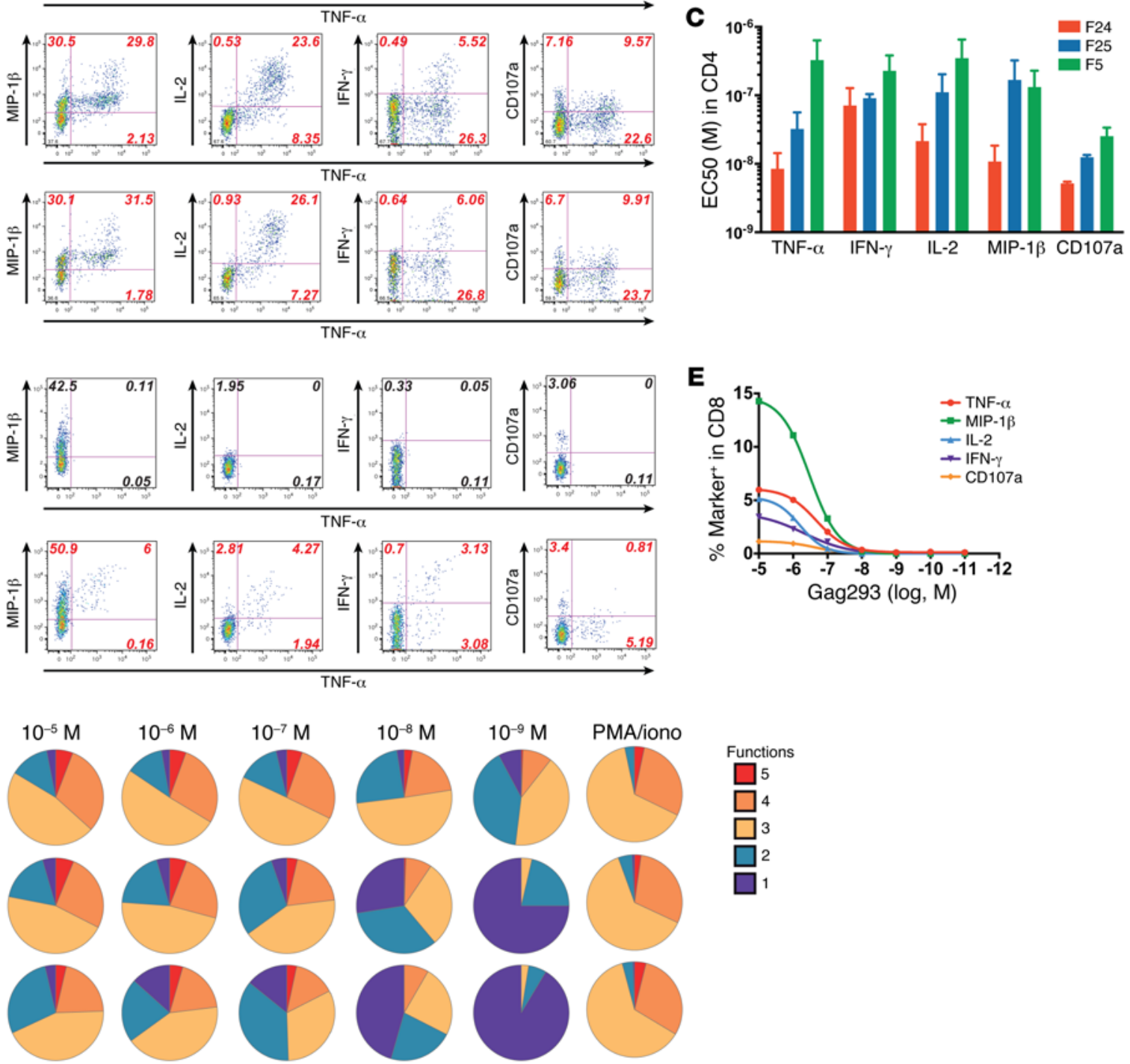

Figure 7. Public TCR transfer confers high-avidity responses and polyfunctionality to primary $\mathrm{T}$ cells. (A) Cytokine production in primary CD4 ${ }^{+} \mathrm{T}$ cells mock transduced or transduced with the F24, F25, or F5 TCR and stimulated with $10^{-5} \mathrm{M}$ Cag293. CD4 ${ }^{+}$T cells were analyzed by ICS for expression of TNF- $\alpha$, MIP-1 $\beta$, IL-2, IFN- $\gamma$, and CD107a. (B) ICS analysis of CD4+ $T$ cells transduced with F24 and stimulated with decreasing Gag293 doses. Expression of the analyzed markers (\% marker+) is reported as a function of peptide dose, after subtraction of background measured in unstimulated cells. (C) Summary of $\mathrm{EC}_{50}$ values measured by ICS in CD4 ${ }^{+} \mathrm{T}$ cells after TCR transduction. For each TCR, the Gag293 concentration required to achieve half-maximal expression of the 5 markers studied is reported. Mean $\pm \mathrm{SEM}$ of $\mathrm{EC}_{50}$ values obtained for 3 independent experiments are reported. (D) Cytokine production in CD8 ${ }^{+} \mathrm{T}$ cells that were mock transduced or transduced with the F24 TCR and analyzed as in A. (E) ICS analysis of CD8 ${ }^{+}$T cells transduced with F24 and stimulated with decreasing Gag293 doses. (F) Polyfunctionality of CD4 ${ }^{+} \mathrm{T}$ cells transduced with the F24, F25, and F5 TCRs and stimulated with decreasing Gag293 doses. The number of coexpressed markers of the 5 studied (TNF- $\alpha$, MIP-1 $\beta$, IL-2, IFN- $\gamma$, CD107a) defines the number of functions reported in legend. Stimulation with PMA/ionomycin was used as a positive control. For $\mathbf{A}, \mathbf{B}, \mathbf{D}, \mathbf{E}$, and $\mathbf{F}, 1$ representative experiment of 3 is shown.
E
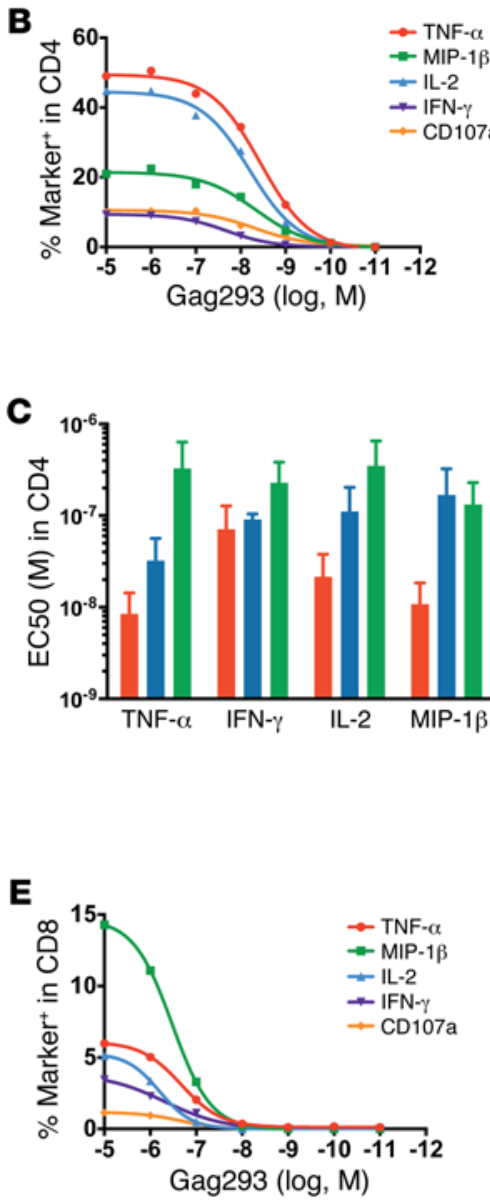

Functions
$\square 5$
$\square_{4}$
$\square_{3}$
$\square_{2}$
$\square 1$ 
in controlled HIV infection, rather than merely the expression of DR11, are associated with high-avidity Gag293-specific responses.

Transfer of the F24 TCR confers polyfunctionality to primary $C D 4^{+}$and $C D 8^{+}$T cells. We next evaluated the properties of the F24, F25, and F5 TCRs when transferred directly into primary T cells, a system physiologically more relevant to potential TCR transfer applications. TCR-transduced PBMCs from healthy donors were analyzed for exogenous TCR expression with a TRBV2-specific mAb. Mock-transduced $\mathrm{CD}^{+} \mathrm{T}$ cells expressed endogenous TRBV2 at levels below 5\%, while TCR-transduced CD4 ${ }^{+} \mathrm{T}$ cells showed TRBV2 expression rates in the $25 \%-35 \%$ range (Supplemental Figure 14A). The hierarchy of HLA-DR tetramer binding was consistent with that observed in J76 cells, with F24 showing significant binding to 4 different tetramers (DR11, DR15, DRB5, and DR1); F25 reacting mostly to the DR11 and DRB5 tetramers and minimally to the DR1 tetramer; and F5 showing barely detectable tetramer binding (Supplemental Figure 14, B and C).

For evaluation of TCR function, PBMCs from DR11, DR15, DR1, or DRB5 donors were TCR transduced and cocultured with autologous MDDCs pulsed with Gag293. A panel of 5 markers, including the cytokines TNF- $\alpha$, IL-2, and IFN- $\gamma$, the chemokine MIP-1 $\beta$ / CCL4, and the degranulation marker CD107a, was assayed by intracellular cytokine staining (ICS). $\mathrm{CD} 4^{+} \mathrm{T}$ cells transduced with the F24, F25, and F5 TCRs showed abundant cytokine production and degranulation capacity after high-dose Gag293 peptide stimulation (Figure 7A). A specific ICS response was detected in $\geq 50 \%$ $\mathrm{CD}^{+} \mathrm{T}$ cells transduced with F24, while in the same experiment TRBV2 expression increased by only $25 \%$ after transduction (Supplemental Figure 14A), pointing to efficient cytokine production even in cells expressing limiting amounts of transduced TCR. ICS responses were then measured in the presence of decreasing Gag293 peptide concentrations (as shown for F24-transduced cells; Figure 7B). The hierarchy of marker induction was conserved across peptide doses, with high expression of TNF- $\alpha$ and IL-2, intermediate expression of MIP-1 $\beta$, and more limited expression of CD107a and IFN- $\gamma$. Of note, specific expression of these 5 markers persisted at very low peptide doses $\left(10^{-9}\right.$ to $\left.10^{-10} \mathrm{M}\right)$. Antigen sensitivity was quantified for the 3 TCRs by measuring the $\mathrm{EC}_{50}$ for induction of the 5 markers tested (Figure 7C). This analysis confirmed the functional hierarchy among the TCRs (F24>F25>F5), which followed the TCR affinity ranking. F24 conferred a highly sensitive detection of Gag293 in primary cells, with $\mathrm{EC}_{50}$ values varying between $10^{-9}$ and $10^{-8} \mathrm{M}$ when TNF- $\alpha$, CD107a, or MIP-1 $\beta$ production was measured. IFN- $\gamma$ responses appeared comparatively less sensitive, with $\mathrm{EC}_{50}$ values between $10^{-8}$ and $10^{-7} \mathrm{M}$, while IL-2 gave intermediate values. Thus, only TCRs with particularly high affinity, such as F24, may lead to full Th1 differentiation with IFN- $\gamma$ production in situations of low antigen availability.

Polyfunctionality, or the capacity to express multiple cytokines simultaneously, is a hallmark of controller T cells and is thought to provide superior effector functions $(16,21,37)$. Quantification of the number of functions (or markers) coexpressed by TCR-transduced cells revealed a high degree of polyfunctionality (Figure $7 F)$. Indeed, the majority ( $>65 \%$ ) of F24-, F25-, and F5-expressing cells could be deemed polyfunctional, as they expressed more than 3 functions after high-dose Gag293 stimulation. CD4 ${ }^{+}$T cells coexpressing the full set of markers ( 5 functions) were detected at peptide doses as low as $10^{-8} \mathrm{M}$ for F24, and as low as $10^{-7} \mathrm{M}$ for F25 and F5. After F24 transfer, polyfunctional cells expressing at least 3 functions still represented more than half of $\mathrm{CD} 4^{+} \mathrm{T}$ cells responding to $10^{-9} \mathrm{M}$ peptide, emphasizing that polyfunctional responses could be achieved after stimulation with minimal amounts of Gag antigen.

Interestingly, $\mathrm{CD}^{+}{ }^{+} \mathrm{T}$ cells transduced with the F24 TCR also showed a Gag293-specific cytokine response, with detectable induction of the 5 markers tested (Figure 7, D and E). Cytokine response in $\mathrm{CD}^{+} \mathrm{T}$ cells transduced with F25 and F5 remained low or undetectable (data not shown). The F24-dependent response in $\mathrm{CD}^{+} \mathrm{T}$ cells could be blocked by treatment with an anti-HLA-DR antibody, indicating that it was restricted by MHC II (Supplemental Figure 15A). Thus, the high-affinity TCR F24 was able to confer a Gag293-specific response in the absence of CD4 coreceptor expression, in a situation where availability of the Lck kinase may be limiting. F24 transfer induced 3 functions or more in greater than $25 \%$ of specific $\mathrm{CD}^{+} \mathrm{T}$ cells at peptide doses as low as $10^{-7} \mathrm{M}$ (Supplemental Figure 15B), indicating that this highaffinity TCR could confer polyfunctional responses to $\mathrm{CD} 8^{+} \mathrm{T}$ cells. Thus, controller clonotypes could be used to generate TCRs with uncommon properties, including affinities in the micromolar range, broad HLA-DR cross-restriction, high antigen sensitivity, and polyfunctionality in both the $\mathrm{CD}^{+}$and $\mathrm{CD} 8^{+} \mathrm{T}$ cell subsets.

\section{Discussion}

Exploration of the TCR repertoire specific for the most immunoprevalent CD4 epitope in HIV-1 Gag revealed a repertoire markedly biased toward the TRAV24 and TRBV2 gene families. Highly prevalent public motifs and public clonotypes were preferentially shared by HIV controllers, suggesting that particular TCR determinants contributed to the efficiency of the antiviral CD4 response in these patients. This notion was reinforced by functional analysis of the most prevalent public clonotypes, as these were able to confer HLA-cross-restricted, highly sensitive, polyfunctional responses against Gag antigens, indicative of superior function. These findings, which to our knowledge provide the first assessment of the HIV-specific $\mathrm{CD}^{+} \mathrm{T}$ cell repertoire at the clonotypic level, emphasize that intrinsic TCR determinants, rather than low antigenemia, specify the remarkable properties of the cellular response in controlled HIV infection.

It was noteworthy that both the TRAV24 and TRBV2 repertoires specific for Gag293 showed a high degree of motif and clonotype sharing. So far, most studies of the human TCR repertoire have focused on TRB genes, but recent analyses suggest that the human TRA repertoire is even more diverse (38). Our findings provide evidence for a dual bias in controlled HIV infection, with both TCR chains showing a significant degree of motif conservation. The most prevalent TRAV24 clonotype (AFKAAGNKLT) was present in 6 of 8 controllers (75\%) studied. In comparison, CD8 TRB public clonotypes reported in previous studies of the HIVspecific repertoire were shared by $2-4$ patients $(13,14,39,40)$. When the analysis was extended to less-stringent definitions of TCR biases, close to half of TRAV24 and TRBV2 clonotypes shared defined public motifs, while the corresponding frequencies were of $29 \%$ and $10 \%$, respectively, for clonotypes of treated patients. Such strong bias in the Gag293-specific TCR repertoire of control- 
lers could not be attributed to amplification of a few clonotypes during culture, as the repertoire of controllers remained highly diverse, with a median of 36 distinct TRBV2 clonotypes per 100 CDR3 sequences. In addition, similar frequencies of public motifs were detected in vitro and ex vivo when the TCR repertoire of controllers could be analyzed in both conditions. Rather, these findings suggest that strong selective pressures (further discussed in the supplemental material) shaped the Gag293-specific repertoire during the establishment of viral control.

The coexpression of TRAV24 and TRBV2 public clonotypes generated functional TCRs that, for some combinations, displayed a remarkably high affinity for Gag293-MHC II complexes. Human TCRs generally show affinities in the 1 to $100 \mu \mathrm{M}$ range by SPR analysis (36). MHC II-restricted TCRs show a trend for a lower affinity as compared with MHC I-restricted TCRs, with average $K_{D}$ values of 70 versus $35 \mu \mathrm{M}$, respectively. With a $K_{D}$ of $0.86 \mu \mathrm{M}$ for the Gag293-DR11 complex, F24 demonstrated one of the highest affinities reported so far for an MHC I- restricted TCR, ranking second in a list of 22 human and mouse TCRs for which structural data are available (36). The molecular determinants of a high-avidity interaction for the Gag293-MHC complex appear to depend both on the TRAV24 and TRBV2 chains and on the restricting HLA allele. The most conserved CDR3 residues in public motifs represent likely candidates for contact residues with Gag293. However, conserved CDR1/CDR2 residues may also contact the Gag293 peptide, considering the predominance of the TRAV24 and TRBV2 families. Future structural studies should help determine the respective roles of the CDR3 versus the CDR1/2 regions in shaping high-avidity interactions for Gag293-MHC complexes. The expression of high-affinity TCRs for Gag293 in controllers resulted in high antigen sensitivity as measured by CD69 induction, with a good correlation between the two parameters $(r=0.85)$. Thus, TCR biophysical properties largely accounted for TCR function, consistent with multiple studies in animal models $(41,42)$. It should be noted, however, that in some studies TCRs with very high or supraphysiological affinities were found to have reduced functions (43). A proposed explanation is that a peptide-MHC (pMHC) that interacts too tightly with a very high-affinity TCR cannot disengage to contact additional TCRs and thus does not achieve serial TCR triggering and T cell response amplification (44). We did not observe decreased functions for the TCR/pMHC II combinations showing the highest affinities by SPR, suggesting that TCR affinities in the order of $1 \mu \mathrm{M}$ are still in the physiological range for optimal TCR function in human $\mathrm{CD}^{+} \mathrm{T}$ cells.

The reasons for the preferential amplification of high-affinity Gag293-specific TCRs in controllers compared with treated patients remain to be elucidated. A particular genetic background or a limited duration of acute infection may have promoted the amplification of such TCRs in controllers (45). Conversely, patients who initially progressed to disease may have lost the high-avidity $\mathrm{CD}^{+} \mathrm{T}$ cell population due to preferential infection and depletion, a notion supported by the restricted CD4 TCR repertoire observed in treated patients, and by rapid loss of certain CD4 specificities after the acute infection stage (6). It will be informative to determine whether other immunoprevalent HIV epitopes elicit a response pattern similar to that observed for
Gag293, with the induction of a highly biased, high-affinity TCR repertoire in controlled infection. The preferential detection of Gag-specific CD4 responses in HIV controllers (20) suggests that several regions of Gag may be targeted by high-affinity TCRs, though this remains to be investigated. High-affinity CD4 TCRs are endowed with strong proliferative capacity (41), which helps explain how they could reach such a dominance in controlled HIV infection. The high affinity of controller TCRs also translated into efficient cytokine secretion upon Gag293 peptide stimulation. Primary CD $4^{+} \mathrm{T}$ cells transduced with the F24 TCR showed $\mathrm{EC}_{50}$ values ranging between $10^{-8} \mathrm{M}$ and $10^{-9} \mathrm{M}$ peptide for the different cytokines studied, indicative of a remarkably high antigen sensitivity for the $\mathrm{CD}^{+} \mathrm{T}$ cell subset. Polyfunctionality, a property that has been associated with HIV control (37), showed a clear dependence on TCR affinity. Comparison of the F24, F25, and F5 TCRs showed that a difference of $1.1 \mathrm{log}$ in affinity resulted in a 2-log difference in the peptide concentration at which half the specific cells retained 3 functions. Thus, TCR affinity appears critical in determining the range of cytokines produced, particularly at low antigen dose. Of interest, not all cytokines were equally dependent on the strength of the TCR signal, with TNF- $\alpha$ showing the lowest requirements and IFN- $\gamma$ the most stringent. The notion of a hierarchy in cytokine production is consistent with a report showing that the affinity of Gag-specific $\mathrm{T}$ cell clones dictated their cytokine expression profile (10). It is relevant that for $\mathrm{CD} 4^{+} \mathrm{T}$ cells, in contrast to $\mathrm{CD}^{+} \mathrm{T}$ cells, IFN- $\gamma$ is the most "demanding" cytokine, as it can be produced only by high-avidity cells upon limiting antigenic stimulation. IFN- $\gamma$ plays a key role in $\mathrm{CD}^{+} \mathrm{T}$ cell helper function, through its capacity to upregulate MHC II in APCs and prime them for efficient antigen presentation. In addition, IFN- $\gamma$ production by tissue $\mathrm{CD} 4^{+} \mathrm{T}$ cells plays an underappreciated role in the recruitment of immune effectors, through the triggering of chemokine cascades (46). In controlled HIV infection, high-avidity Gag-specific $\mathrm{CD} 4^{+} \mathrm{T}$ cells may play a similar role, by keeping the immune system in constant alert and rapidly recruiting $\mathrm{CD}^{+}$ T cells and NK cells to sites of HIV replication upon the occurrence of viral replication blips. In addition, IFN- $\gamma$ secretion may play a direct antiviral role, through the induction of IFN-stimulated genes (ISGs) that inhibit HIV replication (47). For instance, Th1 cells are thought to be less infectable than Th2 cells due to their higher expression of the HIV restriction factor APOBEC3G (48). Highly differentiated Th1 effectors that reexpress CD45RA appear particularly resistant to HIV infection (49). Gag-specific CD4 ${ }^{+} \mathrm{T}$ cells in controllers maintain a Th1 differentiation status with persistent IFN- $\gamma$ production (22), raising the possibility that such cells possess a degree of resistance to HIV infection. This would be consistent with studies that documented a lower susceptibility of controller $\mathrm{CD}^{+}{ }^{+} \mathrm{T}$ cells to HIV replication in vitro (50). Thus, high TCR affinity, with the associated capacity for IFN- $\gamma$ expression at low antigen dose, may contribute to HIV control at several levels, through sensitive immune surveillance, rapid triggering of helper functions, and direct antiviral effector mechanisms.

Of interest, the F24 TCR was able to confer polyfunctional cytokine responses when transferred into $\mathrm{CD}^{+} \mathrm{T}$ cells. The responses were MHC II restricted, indicating that the F24 TCR could interact with the Gag293-MHC II complex in the absence of the CD4 coreceptor. The CD4 molecule is not thought to contrib- 
ute significantly to the affinity of the pMHC/TCR/CD4 complex but plays an important role in relocating the kinase Lck close to the TCR/CD3 signaling complex (51). The lower cytokine responses in $\mathrm{CD}^{+}$than in $\mathrm{CD}^{+} \mathrm{T}$ cells may thus result from a lower number of Lck molecules available for triggering intracellular signals. The transfer of the F24 TCR to $\mathrm{CD}^{+} \mathrm{T}$ cells still conferred the 5 functions tested at the $10^{-5} \mathrm{M}$ to $10^{-7} \mathrm{M}$ antigen dose, suggesting that transduced $\mathrm{CD}^{+} \mathrm{T}$ cells could become efficient effectors in foci of productive HIV replication. These findings open the possibility of reprogramming $\mathrm{CD}^{+} \mathrm{T}$ cells to target the highly conserved major homology region (MHR) of capsid, which could be advantageous given the high fitness cost associated with mutations in this region (52). The report that a CMV-based protective SIV vaccine elicited a high frequency of unconventional MHC II-restricted CD8 responses highlights the potential benefits of $\mathrm{CD}^{+} \mathrm{T}$ cell reprogramming (53). Studies of TCR transfer for cancer immunotherapy have shown that transferring the same TCR into both $\mathrm{CD}^{+}$and $\mathrm{CD}^{+} \mathrm{T}$ cells could enhance tumoricidal activity (54). Similarly, the transfer of a high-avidity Gag-specific TCRs in both $\mathrm{CD}^{+}$and $\mathrm{CD}^{+} \mathrm{T}$ cell populations may be of interest for adoptive $\mathrm{T}$ cell therapies targeting HIV, in order to trigger a full set of antiviral functions.

In conclusion, study of TCRs specific for the immunoprevalent CD4 epitope in capsid revealed that particular clonotypes are associated with HIV control. The TCR repertoire of controllers was characterized by a high prevalence of public TRAV24 and TRBV2 chains. Reconstituted TCRs showed affinities that reached the micromolar range, at the high end of values obtained for naturally expressed TCRs. Public clonotypes conferred MHC II cross-restriction, high antigen sensitivity, and polyfunctionality to $\mathrm{CD}^{+} \mathrm{T}$ cells, suggesting a key role in shaping the properties of an efficient CD4 response. The most prevalent public clonotype also proved functional in $\mathrm{CD}^{+} \mathrm{T}$ cells, suggesting that it could be used to target the highly conserved capsid MHR region in patients of diverse HLA types. Inducing or transferring such clonotypes may contribute to the development of immunotherapeutic approaches that aim at a functional cure of HIV infection.

\section{Methods}

Study design. HIV controllers (HIC group; $n=14$ ) were recruited through the CO21 CODEX cohort implemented by ANRS. HIV controllers were defined as HIV-1-infected patients who had been seropositive for more than 5 years, had received no antiretroviral treatment, and for whom more than $90 \%$ of plasma viral load measurements were undetectable by standard assays. All HIV controllers included in the study had current viral loads of less than 50 copies $/ \mathrm{ml}$. The group of efficiently treated patients (HAART group; $n=14$ ) had received antiretroviral therapy for a minimum of 5 years and showed long term HIV-1 suppression with viral loads of less than 50 copies $/ \mathrm{ml}$. Treated patients were recruited at the Raymond Poincaré and Bicêtre hospitals (France). Patients were included in the TCR study if their genotype matched at least one of the following alleles: $D R B 1^{*} 0101$ (DR1), DRB1*1101 (DR11), DRB1*1501 (DR15), or DRB5*0101 (DRB5). Healthy donors were anonymous volunteers who donated blood at Etablissement Français du Sang.

Molecular and functional TCR analysis. Molecular and functional analyses of the Gag293-specific TCR repertoire are described in Supplemental Methods.
Statistics. Statistics were computed with Prism version 6.0 software (GraphPad) and R version 3.2.3 software (https://www.r-project. org/). $P$ values less than 0.05 were considered statistically significant. Differences between groups were analyzed with the nonparametric Mann-Whitney $U$ test, with the exception of total clonotypic repertoires, for which means were compared by unpaired 2-tailed $t$ tests. Differences in cell line response frequencies and HLA-DR allele frequencies were analyzed in contingency tables with Fisher's exact test. Differences in proportions of CDR3 lengths were computed with a 2-sample test for equality of proportions with continuity correction R. Correlations were analyzed with the nonparametric Spearman's coefficient. $\mathrm{EC}_{50}$ values were obtained after non-linear curve fit using a sigmoidal dose response model in Prism. All significant differences between groups $(P<0.05)$ were reported in data plots.

Study approval. The study was promoted by ANRS and approved by the Comité de Protection des Personnes Ile-de-France VII under number 05-22. All participants gave written informed consent prior to inclusion in the study.

\section{Author contributions}

DB, MG, OL, SG, AL, MM, AN, KAC, BL, MC, PDT, JR, JFD, FAS, and LAC designed and/or performed experiments. DB, MG, SG, $\mathrm{AL}, \mathrm{SH}, \mathrm{PL}, \mathrm{FB}$, and LAC analyzed data. DB, MG, and LAC wrote the manuscript. OL, SG, JR, and FA edited the manuscript.

\section{Acknowledgments}

This study was carried out in the framework of the ANRS EP36 HIV Controllers Study Group. We are grateful to the patients who participated in the study. We thank the clinicians who recruited patients for this study: Huguette Berthé, David Zucman, Dominique Bornarel, Olivier Bouchaud, Patricia Honoré, Philippe Genet, Juliette Gerbe, Olivier Patey, Laurent Richier, Katia Bourdic, Pierre-Marie Girard, Bénédicte Lefebvre, Michèle Pauchard, Jean-Michel Molina, Sylvie Parlier, Caroline Lascaux-Combe, Samuel Ferret, Valérie Garrait, and Isabelle DeLacroix-Szmania. We thank Pierre Charneau, Mirjam Heemskerk, Fabrice Lemaître, Bernard Maillère, Christiane Moog, Olivier Schwartz, and Philippe Souque for advice and reagents; the team of the Pasteur Center for Human Immunology for help with cell sorting; and the team of the NIH Tetramer Core facility at Emory University for reagents and advice on tetramer loading. This work was supported by ANRS, Sidaction, the Pasteur Institute, the Australian Research Council (ARC), and the National Health and Medical Research Council of Australia (NHMRC). L.A. Chakrabarti is supported by grants grants from ANRS (EP36-8) and Agence Nationale de la Recherche (ANR PD1VAX); S. Gras is supported by an ARC Future Fellowship (FF120100416), and J. Rossjohn by an NHMRC Australia Fellowship (AF50). D. Benati was the recipient of postdoctoral fellowships from ANRS, the Fondation pour la Recherche Médicale, and the Institut Servier. M. Galperin is the recipient of an ANRS doctoral fellowship. M. Mukhopadhyay is a scholar in the Pasteur - Paris University (PPU) International PhD program.

Address correspondence to: Lisa A. Chakrabarti or Daniela Benati, Unité de Pathogénie Virale, Institut Pasteur, 28 rue du Dr Roux, 75724 Paris Cedex 15, France. Phone: 33.1.45.68.89.45; E-mail: chakra@pasteur.fr (L.A. Chakrabarti ), daniela.benati@ unimore.it (D. Benati). 
1. Deeks SG, et al. Systemic effects of inflammation on health during chronic HIV infection. Immunity. 2013;39(4):633-645.

2. Connors M, et al. HIV infection induces changes in $\mathrm{CD}_{4}^{+} \mathrm{T}$-cell phenotype and depletions within the $\mathrm{CD} 4^{+} \mathrm{T}$-cell repertoire that are not immediately restored by antiviral or immune-based therapies. Nat Med.1997;3(5):533-540.

3. Gorochov G, et al. Perturbation of $\mathrm{CD} 4^{+}$and $\mathrm{CD} 8$ $\mathrm{T}$-cell repertoires during progression to AIDS and regulation of the $\mathrm{CD} 4^{+}$repertoire during antiviral therapy. Nat Med.1998;4(2):215-221.

4. Rosenberg ES, et al. Vigorous HIV-1-specific $\mathrm{CD} 4^{+} \mathrm{T}$ cell responses associated with control of viremia. Science. 1997;278(5342):1447-1450.

5. Douek DC, et al. HIV preferentially infects HIV-specific $\mathrm{CD} 4^{+} \mathrm{T}$ cells. Nature. 2002;417(6884):95-98.

6. Lubong Sabado R, et al. In vitro priming recapitulates in vivo HIV-1 specific $\mathrm{T}$ cell responses, revealing rapid loss of virus reactive $\mathrm{CD} 4$ T cells in acute HIV-1 infection. PLoS One. 2009;4(1):e4256.

7. Lambotte O, et al. HIV controllers: a homogeneous group of HIV-1-infected patients with spontaneous control of viral replication. Clin Infect Dis. 2005;41(7):1053-1056.

8. Chakrabarti LA, Simon V. Immune mechanisms of HIV control. Curr Opin Immunol. 2010;22(4):488-496.

9. Saez-Cirion A, et al. HIV controllers exhibit potent CD8 $\mathrm{T}$ cell capacity to suppress HIV infection ex vivo and peculiar CTL activation phenotype. Proc Natl Acad Sci U S A. 2007;104(16):6776-6781.

10. Almeida JR, et al. Antigen sensitivity is a major determinant of $\mathrm{CD} 8^{+} \mathrm{T}$-cell polyfunctionality and HIV-suppressive activity. Blood. 2009;113(25):6351-6360.

11. Ladell $\mathrm{K}$, et al. A molecular basis for the control of preimmune escape variants by HIV-specific CD8 ${ }^{+}$T cells. Immunity. 2013;38(3):425-436.

12. Chen $\mathrm{H}$, et al. TCR clonotypes modulate the protective effect of HLA class I molecules in HIV-1 infection. Nat Immunol. 2012;13(7):691-700.

13. Almeida JR, et al. Superior control of HIV-1 replication by $\mathrm{CD} 8^{+} \mathrm{T}$ cells is reflected by their avidity, polyfunctionality, and clonal turnover. J Exp Med. 2007;204(10):2473-2485.

14. Iglesias MC, et al. Escape from highly effective public CD8+ T-cell clonotypes by HIV. Blood. 2011;118(8):2138-2149.

15. Younes SA, et al. HIV-1 viremia prevents the establishment of interleukin 2-producing HIV-specific memory $\mathrm{CD} 4^{+} \mathrm{T}$ cells endowed with proliferative capacity. J Exp Med. 2003;198(12):1909-1922.

16. Harari A, et al. Functional heterogeneity of memory CD4 $\mathrm{T}$ cell responses in different conditions of antigen exposure and persistence. J Immunol. 2005;174(2):1037-1045.

17. van Grevenynghe J, et al. Transcription factor FOXO3a controls the persistence of memory CD4(+) T cells during HIV infection. Nat Med. 2008;14(3):266-274.

18. Potter SJ, et al. Preserved central memory and activated effector memory $\mathrm{CD} 4^{+} \mathrm{T}$-cell subsets in human immunodeficiency virus controllers: an ANRS EP36 study. J Virol. 2007;81(24):13904-13915.

19. Kaufmann DE, et al. Limited durability of viral control following treated acute HIV infection. PLoS Med. 2004;1(2):e36.

20. Ranasinghe $\mathrm{S}$, et al. HIV-specific CD4 T cell responses to different viral proteins have discordant associations with viral load and clinical outcome. J Virol. 2012;86(1):277-283.

21. Ferre AL, et al. HIV controllers with HLADRB1 ${ }^{*} 13$ and HLA-DQB1 ${ }^{*} 06$ alleles have strong, polyfunctional mucosal $\mathrm{CD} 4^{+} \mathrm{T}$-cell responses. JVirol.2010;84(21):11020-11029.

22. Vingert B, et al. HIV controllers maintain a population of highly efficient Th1 effector cells in contrast to patients treated in the long term. J Virol. 2012;86(19):10661-10674.

23. Kaufmann DE, et al. Upregulation of CTLA- 4 by $\mathrm{HIV}$-specific $\mathrm{CD} 4^{+} \mathrm{T}$ cells correlates with disease progression and defines a reversible immune dysfunction. Nat Immunol. 2007;8(11):1246-1254.

24. Porichis F, et al. Responsiveness of HIVspecific CD4 T cells to PD-1 blockade. Blood. 2011;118(4):965-974.

25. Vingert B, et al. HIV controller $\mathrm{CD}^{+}{ }^{+} \mathrm{T}$ cells respond to minimal amounts of Gag antigen due to high TCR avidity. PLoS Pathog. 2010;6(2):e1000780.

26. Alexander-Miller MA. High-avidity $\mathrm{CD} 8^{+} \mathrm{T}$ cells: optimal soldiers in the war against viruses and tumors. Immunol Res. 2005;31(1):13-24.

27. Berger CT, et al. High functional avidity CTL responses to HLA-B-restricted Gag-derived epitopes associate with relative HIV control. JVirol. 2011;85(18):9334-9345.

28. Kaufmann DE, et al. Comprehensive analysis of human immunodeficiency virus type 1-specific CD4 responses reveals marked immunodominance of gag and nef and the presence of broadly recognized peptides. J Virol. 2004;78(9):4463-4477.

29. Wooldridge L, et al. Tricks with tetramers: how to get the most from multimeric peptide-MHC. Immunology. 2009;126(2):147-164.

30. Lim A, et al. Frequent contribution of T cell clonotypes with public TCR features to the chronic response against a dominant EBV-derived epitope: application to direct detection of their molecular imprint on the human peripheral $\mathrm{T}$ cell repertoire. JImmunol. 2000;165(4):2001-2011.

31. Hacein-Bey-Abina S, et al. Efficacy of gene therapy for X-linked severe combined immunodeficiency. N EnglJMed. 2010;363(4):355-364.

32. Alamyar E, et al. IMGT((R)) tools for the nucleotide analysis of immunoglobulin (IG) and T cell receptor (TR) V-(D)-J repertoires, polymorphisms, and IG mutations: IMGT/V-QUEST and IMGT/HighV-QUEST for NGS. Methods Mol Biol. 2012;882:569-604

33. Venturi V, et al. A mechanism for TCR sharing between $T$ cell subsets and individuals revealed by pyrosequencing. JImmunol. 2011;186(7):4285-4294

34. Turner SJ, et al. Structural determinants of T-cell receptor bias in immunity. Nat Rev Immunol. 2006;6(12):883-894.

35. Gras S, et al. T-cell receptor bias and immunity. Curr Opin Immunol. 2008;20(1):119-125.

36. Rossjohn J, et al. T cell antigen receptor recognition of antigen-presenting molecules. Anпи Rev Immunol. 2015;33:169-200.

37. Betts MR, et al. HIV nonprogressors preferentially maintain highly functional HIV-specific CD8 ${ }^{+}$T cells. Blood. 2006;107(12):4781-4789.

38. Liu P, et al. Characterization of human alphabetaTCR repertoire and discovery of D-D fusion in TCRbeta chains. Protein Cell. 2014;5(8):603-615.

39. Gillespie GM, et al. Strong TCR conservation and altered $\mathrm{T}$ cell cross-reactivity characterize a $\mathrm{B}^{*} 57$ restricted immune response in HIV-1 infection. JImmunol. 2006;177(6):3893-3902.

40. Kloverpris HN, et al. CD8 ${ }^{+}$TCR bias immunodominance in HIV-1 infection. JImmunol. 2015;194(11):5329-5345.

41. Williams MA, et al. Rapid culling of the $\mathrm{CD}_{4}^{+} \mathrm{T}$ cell repertoire in the transition from effector to memory. Immunity. 2008;28(4):533-545.

42. Belyakov IM, et al. Impact of vaccine-induced mucosal high-avidity $\mathrm{CD} 8^{+} \mathrm{CTLs}$ in delay of AIDS viral dissemination from mucosa. Blood. 2006;107(8):3258-3264.

43. Corse $\mathrm{E}$, et al. Attenuated $\mathrm{T}$ cell responses to a high-potency ligand in vivo. PLoS Biol. 2010;8(9):e1000481.

44. Valitutti $S$. The serial engagement model 17 years after: from TCR triggering to immunotherapy. Front Immunol. 2012;3:272.

45. Thorborn G, et al. Clonotypic composition of the $\mathrm{CD} 4^{+} \mathrm{T}$ cell response to a vectored retroviral antigen is determined by its speed. J Immunol. 2014;193(4):1567-1577.

46. Swain SL, et al. Expanding roles for CD4(+) T cells in immunity to viruses. Nat Rev Immunol. 2012;12(2):136-148

47. Blanco-Melo D, et al. Intrinsic cellular defenses against human immunodeficiency viruses. Immunity. 2012;37(3):399-411.

48. Vetter ML, et al. Differences in APOBEC3G expression in $\mathrm{CD}^{+} \mathrm{T}$ helper lymphocyte subtypes modulate HIV-1 infectivity. PLoS Pathog. 2009;5(2):e1000292.

49. Oswald-Richter K, et al. Identification of a CCR5-expressing $\mathrm{T}$ cell subset that is resistant to R5-tropic HIV infection. PLoS Pathog. 2007;3(4):e58.

50. Saez-Cirion A, et al. Restriction of HIV-1 replication in macrophages and $\mathrm{CD} 4^{+} \mathrm{T}$ cells from HIV controllers. Blood. 2011;118(4):955-964.

51. Reinherz EL, Wang JH. Codification of bidentate pMHC interaction with TCR its co-receptor. Trends Immunol. 2015;36(5):300-306.

52. Rihn SJ, et al. Extreme genetic fragility of the HIV-1 capsid. PLoS Pathog. 2013;9(6):e1003461.

53. Hansen SG, et al. Cytomegalovirus vectors violate $\mathrm{CD} 8^{+} \mathrm{T}$ cell epitope recognition paradigms. Science. 2013;340(6135):1237874.

54. Ghorashian S, et al. CD8 T cell tolerance to a tumor-associated self-antigen is reversed by CD4 T cells engineered to express the same T cell receptor. J Immunol. 2015;194(3):1080-1089. 